\title{
STEADY-STATE AND PERIODIC EXPONENTIAL TURNPIKE PROPERTY FOR OPTIMAL CONTROL PROBLEMS IN HILBERT SPACES*
}

\author{
EMMANUEL TRÉLAT ${ }^{\dagger}$, CAN ZHANG $^{\ddagger}$, AND ENRIQUE ZUAZUA§
}

\begin{abstract}
In this work, we study the steady-state (or periodic) exponential turnpike property of optimal control problems in Hilbert spaces. The turnpike property, which is essentially due to the hyperbolic feature of the Hamiltonian system resulting from the Pontryagin maximum principle, reflects the fact that, in large control time horizons, the optimal state and control and adjoint state remain most of the time close to an optimal steady-state. A similar statement holds true as well when replacing an optimal steady-state by an optimal periodic trajectory. To establish the result, we design an appropriate dichotomy transformation, based on solutions of the algebraic Riccati and Lyapunov equations. We illustrate our results with examples including linear heat and wave equations with periodic tracking terms.
\end{abstract}

Key words. exponential turnpike property, periodic tracking, periodic optimal controls, stability analysis, dichotomy transformation

AMS subject classifications. 49J20, 49K20, 93D20

DOI. $10.1137 / 16 \mathrm{M} 1097638$

1. Introduction. The turnpike property of optimal trajectories was first observed and investigated by economists for finite-dimensional discrete-time optimal control problems (see, e.g., [25]). The turnpike property reflects the fact that, for an optimal control problem for which the time horizon is large enough, its optimal solution remains most of the time close to a referred turnpike, which is usually the optimal solution of a corresponding "static" optimal control problem. In the last decades, several turnpike theorems for optimal control problems have been obtained in a large number of works (see, for instance, $[1,3,7,8,9,10,11,14,16,23,25,27$, $28,30,37,46,47,48,51]$ and references therein) for discrete-time or continuous-time problems involving control systems in finite dimension.

The usual turnpike property is somehow a qualitative feature for the limiting structure of optimal solutions to the optimal control problem as the time horizon tends to infinity. The exponential turnpike property is a quantitative version (see,

*Received by the editors October 6, 2016; accepted for publication (in revised form) December 26, 2017; published electronically March 29, 2018.

http://www.siam.org/journals/sicon/56-2/M109763.html

Funding: The authors acknowledge the financial support by the grant FA9550-14-1-0214 of the EOARD-AFOSR. The second author was partially supported by the National Natural Science Foundation of China under grants 11501424 and 11371285. The third author was partially supported by the Advanced Grant DYCON (Dynamic Control) of the European Research Council Executive Agency, FA9550-15-1-0027 of AFOSR, the MTM2014-52347 and MTM2017-92996 grants of the MINECO (Spain), and ICON of the French ANR.

†Sorbonne Université, Université Paris-Diderot SPC, CNRS, Inria, Laboratoire Jacques-Louis Lions, équipe CAGE, F-75005 Paris, France (emmanuel.trelat@upmc.fr).

${ }^{\ddagger}$ School of Mathematics and Statistics, Wuhan University, 430072 Wuhan, China; Sorbonne Université, Université Paris-Diderot SPC, CNRS, Inria, Laboratoire Jacques-Louis Lions, F-75005 Paris, France (zhangcansx@163.com).

$\S^{\S}$ DeustoTech, Fundación Deusto, Avda Universidades, 24, 48007, Bilbao, Basque Country, Spain; Departamento de Matemáticas, Universidad Autónoma de Madrid, 28049 Madrid, Spain; Facultad Ingeniería, Universidad de Deusto, Avda. Universidades, 24, 48007 Bilbao, Basque Country, Spain; Sorbonne Université, Université Paris-Diderot SPC, CNRS, Inria, Laboratoire Jacques-Louis Lions, F-75005, Paris, France (enrique.zuazua@uam.es). 
e.g., $[9,27,37])$ ensuring that the optimal solution remains exponentially close to the referred turnpike for a sufficiently large time interval contained in the time horizon frame. In a different context, the authors of [6] proved that the long-time averages of the solution for a mean field game system converge exponentially to the solution of the associated stationary ergodic mean field game.

In the recent work [27], the exponential turnpike property has been established for linear quadratic optimal control problems in finite dimension under the Kalman controllability rank condition, as well as for linear infinite-dimensional systems, including the cases of linear heat and wave equations with internal controls under some observability inequality assumptions. A local version, for semilinear heat equations, has been obtained in [28], under a smallness condition on the target to be tracked.

The authors of [37] established the exponential turnpike property for general finite-dimensional nonlinear control systems with general terminal constraint conditions, under some appropriate controllability and smallness assumptions. The main ingredient in [37] is an exponential dichotomy transformation early established in [43] to uncouple the two-point boundary value problem coming from the Pontryagin maximum principle, reflecting the hyperbolicity feature of the Hamiltonian system.

In $[27,28,37]$, not only the optimal state and control, but also the corresponding adjoint vector, resulting from the application of the Pontryagin maximum principle, were shown to remain exponentially close to an extremal triple for the corresponding static optimal control problem, except at the extremities of the time frame. The latter property is particularly useful in order to implement and initialize successfully a numerical shooting method for solving the optimality system (see [37]).

The objective of the present paper is to establish the exponential turnpike property for general infinite-dimensional nonlinear optimal control problems under exponential stabilizability and detectability assumptions, as well as some smallness assumptions. This extends to an abstract Hilbert space setting the main results in [28] and [37]. The result implies that, except at the beginning and at the end of the time frame, the optimal trajectory remains exponentially close to a steady-state, which is itself characterized as being a minimizer for the associated "static optimal control problem."

As a second main result, we consider linear quadratic optimal control problems with periodic tracking trajectories, i.e., linear autonomous control systems (still in Hilbert spaces) with a quadratic cost in which the integrand involves a periodic tracking term. We prove that, under exponential stabilizability and detectability assumptions, the optimal trajectory (also, control and adjoint state) remains exponentially close, except at the beginning and the end of the time frame, to a periodic optimal trajectory, which is characterized as being the optimal solution of an associated periodic optimal control problem. We are not aware of any general result establishing such a periodic exponential turnpike property, even in the finite-dimensional case. Note however that Samuelson established in [34] a periodic turnpike property for a specific optimal growth problem in economics in which the integrand of the minimization functional is periodic. Periodic turnpike has been also considered in the recent paper [45] within the dissipativity context.

To prove the results, our approach takes advantage of the hyperbolic feature of the optimality systems (see [32]) resulting from the Pontryagin maximum principle, as in [37]. However, the invertibility of solutions of the matrix algebraic Riccati equation played an important role in the argument of [37], but to the best of our knowledge, this argument is in general not valid in the infinite-dimensional setting, because this invertibility is closely related to an exact observability inequality and thus this would 
be a too restrictive assumption in view of applications. One of the main technical novelties of the present paper is to design a new dichotomy transformation to overcome this difficulty. We mentioned that the authors in [27] have used the Riccati theory, although slightly different, to describe the evolution of the optimality system as the time horizon tends to infinity.

The paper is organized in the following way. In section 2 , we present our main results and some applications. More precisely, in Theorem 1, we establish the exponential turnpike property for general nonlinear autonomous optimal control problems with bounded control operators in a Hilbert space, under appropriate stability assumptions and smallness conditions. In Theorem 2, we establish the periodic exponential turnpike property for linear quadratic optimal control problems with bounded control operators, in which the referred turnpike is a periodic optimal solution for a periodic optimal control problem. In Theorems 4 and 5, we extend the results, respectively, of Theorems 1 and 2 to analytic parabolic systems with unbounded admissible control operators. Section 3 is devoted to the proofs of the main results.

2. Main results. Throughout the paper, given a Hilbert space $Z$, we denote by $\langle\cdot, \cdot\rangle_{Z}$ the usual inner product and by $\|\cdot\|_{Z}$ the corresponding norm. The notation $L(X, Y)$ designates the space of bounded linear operators from the Hilbert space $X$ to the Hilbert space $Y$.

2.1. Steady-state exponential turnpike for nonlinear optimal control problems. Let $X$ and $U$ be two Hilbert spaces, which are accordingly identified with their duals. We define hereafter the dynamical optimal control problem $\left(O C P^{T}\right)$, and then the corresponding static optimal control problem $\left(P_{s}\right)$ yielding the optimal steady-state around which the turnpike is expected. The exponential turnpike result is then stated in Theorem 1.

The dynamical optimal control problem $\left(O C P^{T}\right)$. For every $T>0$ and every $y_{0} \in X$, we consider the optimal control problem

$$
\left(O C P^{T}\right) \quad \inf _{u(\cdot) \in L^{2}(0, T ; U)} J^{T}(u(\cdot))=\int_{0}^{T} f^{0}(y(t), u(t)) d t,
$$

where $y(\cdot) \in C([0, T] ; X)$ is the mild solution ${ }^{1}$ of

$$
\left\{\begin{array}{l}
\dot{y}(t)=A y(t)+f(y(t), u(t)) \text { for a.e. } t \in[0, T] \\
y(0)=y_{0}
\end{array}\right.
$$

corresponding to the control function $u(\cdot) \in L^{2}(0, T ; U)$. Here, $A: D(A) \subset X \rightarrow X$ is a linear (unbounded) operator generating a $C_{0}$ semigroup on $X$, and the function $f^{0}: X \times U \rightarrow \mathbb{R}$ and the mapping $f: X \times U \rightarrow X$ are assumed to be twice continuously Fréchet differentiable and globally Lipschitz continuous with respect to $y$ for each $u \in U$.

Existence of optimal controls for the problem $\left(O C P^{T}\right)$ is a classical issue (see, e.g., [24, Chapter 3]) and is generally ensured under adequate convexity assumptions. Here, we assume that for the problem $\left(O C P^{T}\right)$ there exists at least one optimal solution. Let $\left(y^{T}(\cdot), u^{T}(\cdot)\right)$ be any of them. According to the Pontryagin maximum principle in a Hilbert space (see [24, Chapter 4]), there exists $\lambda^{T}(\cdot) \in C([0, T], X)$,

\footnotetext{
${ }^{1}$ Recall that the form of mild solution is $y(t)=e^{A t} y_{0}+\int_{0}^{t} e^{A(t-s)} f(y(s), u(s)) d s, t \in[0, T]$, where $e^{A t}$ is the $C_{0}$ semigroup in $X$ with generator $A: D(A) \subset X \rightarrow X$.
} 
called adjoint state or costate, such that

$$
\begin{cases}\dot{y}^{T}(t)=A y^{T}(t)+H_{\lambda}\left(y^{T}(t), \lambda^{T}(t), u^{T}(t)\right), & y^{T}(0)=y_{0}, \\ \dot{\lambda}^{T}(t)=-A^{*} \lambda^{T}(t)-H_{y}\left(y^{T}(t), \lambda^{T}(t), u^{T}(t)\right), & \lambda^{T}(T)=0,\end{cases}
$$

in the mild sense along $[0, T]$ and

$$
H_{u}\left(y^{T}(t), \lambda^{T}(t), u^{T}(t)\right)=0 \text { for a.e. } t \in[0, T],
$$

where $A^{*}$ is the adjoint operator associated with $A$, with the domain $D\left(A^{*}\right)$, and

$$
H(y, \lambda, u)=\langle\lambda, f(y, u)\rangle_{X}-f^{0}(y, u)
$$

is the Hamiltonian of the optimal control problem. The index at $H$ above designates the partial derivative.

The static optimal control problem $\left(P_{s}\right)$. We consider the nonlinear constrained minimization problem

$$
\left(P_{s}\right) \quad \inf _{u \in U} J_{s}(u)=f^{0}(y, u)
$$

where $y \in X$ is the corresponding weak solution ${ }^{2}$ of

$$
A y+f(y, u)=0 .
$$

Likewise, we assume that the problem $\left(P_{s}\right)$ has at least one optimal solution (sufficient conditions ensuring existence are standard; see, e.g., [17]). Let $\left(y_{s}, u_{s}\right) \in X \times U$ be any of them. We assume that there exists $\lambda_{s} \in X$ such that $\left(y_{s}, \lambda_{s}, u_{s}\right)$ satisfies the optimality system (see, e.g., [24, Chapter 5, Theorem 1.2] and $[17,35]$ )

$$
\left\{\begin{array}{r}
A y_{s}+H_{\lambda}\left(y_{s}, \lambda_{s}, u_{s}\right)=0 \\
-A^{*} \lambda_{s}-H_{y}\left(y_{s}, \lambda_{s}, u_{s}\right)=0
\end{array}\right.
$$

and

$$
H_{u}\left(y_{s}, \lambda_{s}, u_{s}\right)=0
$$

Here, $H$ is the Hamiltonian function defined by (2.4).

Note that $\left(y_{s}, \lambda_{s}, u_{s}\right)$ is an equilibrium point of the differential system (2.2), satisfying the constraint (2.3). This remark is crucial in order to understand the turnpike property. Indeed, we are going to prove that, under appropriate assumptions, the equilibrium point $\left(y_{s}, \lambda_{s}, u_{s}\right)$ is hyperbolic, in the sense that, if we linearize the system of equations (2.2) around the point $\left(y_{s}, \lambda_{s}, u_{s}\right)$, then we obtain a linear system that has a stable manifold and an unstable manifold. This feature, adequately interpreted, implies the exponential turnpike property, locally around $\left(y_{s}, \lambda_{s}, u_{s}\right)$.

The exponential turnpike property. At the point $\left(y_{s}, \lambda_{s}, u_{s}\right)$, we denote by

$$
H_{\lambda y}=H_{\lambda y}\left(y_{s}, \lambda_{s}, u_{s}\right), \quad H_{\lambda u}=H_{\lambda u}\left(y_{s}, \lambda_{s}, u_{s}\right), \quad H_{u u}=H_{u u}\left(y_{s}, \lambda_{s}, u_{s}\right)
$$

and

$$
H_{u y}=H_{u y}\left(y_{s}, \lambda_{s}, u_{s}\right), \quad H_{y u}=H_{y u}\left(y_{s}, \lambda_{s}, u_{s}\right), \quad H_{y y}=H_{y y}\left(y_{s}, \lambda_{s}, u_{s}\right) .
$$

\footnotetext{
${ }^{2}$ Recall that the form of weak solution $y \in X$ is $\left\langle y, A^{*} \varphi\right\rangle_{X}+\langle f(y, u), \varphi\rangle_{X}=0, \quad$ for any $\varphi \in$ $D\left(A^{*}\right)$.
} 
Then, we define

$$
\mathcal{A}=A+H_{\lambda y}-H_{\lambda u} H_{u u}^{-1} H_{u y}
$$

whenever $H_{u u}^{-1}$ exists.

THEOREM 1. Assume that $H_{u u}$ is negative definite and boundedly invertible ${ }^{3}$ and that $H_{y u} H_{u u}^{-1} H_{u y}-H_{y y}$ is positive semidefinite, i.e.,

$$
H_{y u} H_{u u}^{-1} H_{u y}-H_{y y}=\mathcal{C}^{*} \mathcal{C} \text { for some } \mathcal{C} \in L(X, X) .
$$

Assume that the pair $\left(\mathcal{A}, H_{\lambda u}\right)$ is exponentially stabilizable ${ }^{4}$ and that the pair $(\mathcal{A}, \mathcal{C})$ is exponentially detectable. ${ }^{5}$ Then, there exist positive constants $\varepsilon, \mu$, and $c$ such that for any $T>0$, if

$$
\left\|y_{0}-y_{s}\right\|_{X}+\left\|\lambda_{s}\right\|_{X} \leqslant \varepsilon
$$

any optimal extremal triple $\left(y^{T}(\cdot), u^{T}(\cdot), \lambda^{T}(\cdot)\right)$ of $\left(O C P^{T}\right)$ has the exponential turnpike property

$$
\left\|y^{T}(t)-y_{s}\right\|_{X}+\left\|u^{T}(t)-u_{s}\right\|_{U}+\left\|\lambda^{T}(t)-\lambda_{s}\right\|_{X} \leqslant c\left(e^{-\mu t}+e^{-\mu(T-t)}\right)
$$

for every $t \in[0, T]$.

Remark 1. The above theorem extends the result established in [37] for general finite-dimensional optimal control problems. It is as well of local nature, requiring the smallness assumptions (2.8) and (2.9). Note that, in Example 1 below, to ensure (2.8) the turnpike itself has to be small. Ruling out the assumption (2.9) would require one to have a knowledge of global properties of the dynamics.

We mention that the authors of [6] have derived a global exponential convergence result, by using a property that the solution of a mean field game system fulfills a smallness property on an interval of the form $(\delta T,(1-\delta) T)$ for $\delta \in(0,1)$ (see $[6$, Lemma 3.6]). However, we cannot prove a similar property to [6, Lemma 3.6] for the optimality system under the global Lipschitz condition of the control system.

Remark 2. As seen in the proof, the exponential decay constant $\mu$ in (2.10) can be characterized as the exponential stability rate for a $C_{0}$ semigroup resulting from the operator algebraic Riccati equation

$$
\mathcal{A}^{*} \mathcal{P}+\mathcal{P} \mathcal{A}+\mathcal{C}^{*} \mathcal{C}+\mathcal{P} H_{\lambda u} H_{u u}^{-1} H_{u \lambda} \mathcal{P}=0 .
$$

The constant $c$ in (2.10) depends in a linear way on $\left(\left\|y_{0}-y_{s}\right\|_{X}+\left\|\lambda_{s}\right\|_{X}\right)$.

Remark 3. Note that we assume that $f$ and $f^{0}$ are $C^{2}$-smooth and globally Lipschitz with respect to the state variable. Under such a globally Lipschitz condition, we would get the existence and uniqueness of solutions for a given Cauchy problem

\footnotetext{
${ }^{3}$ This assumption is standard in optimal control theory, and it is usually referred as a strong Legendre condition; see, e.g., [36]. It implies that the optimal control can be locally represented by the maximum condition in terms of the optimal state and adjoint state.

${ }^{4}$ The pair $\left(\mathcal{A}, H_{\lambda u}\right)$ is said to be exponentially stabilizable if and only if there exists an operator $\mathcal{K} \in L(X, U)$ such that the operator $\mathcal{A}+H_{\lambda u} \mathcal{K}$ is exponentially stable, i.e., the operator $\mathcal{A}+H_{\lambda u} \mathcal{K}$ generates a $C_{0} \operatorname{semigroup}(\mathcal{S}(t))_{t \geqslant 0}$ satisfying $\|\mathcal{S}(t)\|_{L(X, X)} \leqslant c e^{-\nu t}$ for all $t \geqslant 0$, for some $c>0$ and $\nu>0$.

${ }^{5}$ The pair $(\mathcal{A}, \mathcal{C})$ is said to be exponentially detectable if $\left(\mathcal{A}^{*}, \mathcal{C}^{*}\right)$ is exponentially stabilizable.
} 
(2.1). The $C^{2}$-regularity of the dynamic seems to be necessary to ensure that we can obtain the linearized system of the optimality systems resulting from the Pontryagin maximum principle for $\left(O C P^{T}\right)$. Although sometimes globally Lipschitz properties are not satisfied, we can however reduce the problem to the globally Lipschitz situation if solutions under consideration remain in a bounded set and if the dynamics and their derivatives are bounded on bounded sets. Then, similar exponential turnpike results can be also established in each particular instance. As an example of Theorem 1, we consider the cubic semilinear heat equation in Example 1, though for which the exponential turnpike property has already been established in [28].

Let us describe how Theorem 1 yields the local exponential turnpike property for the semilinear heat equation in [28].

Example 1 . Let $\Omega \subset \mathbb{R}^{3}$ be an open and bounded domain with a $C^{2}$ boundary, and let $\omega \subset \Omega$ be a nonempty open subset. Denote by $\chi_{\omega}$ the characteristic function of the subset $\omega$. Given $T>0, y_{d} \in L^{2}(\Omega)$, and $y_{0} \in L^{2}(\Omega)$, we consider the optimal control problem

$$
\text { Minimize } \frac{1}{2} \int_{0}^{T} \int_{\Omega}\left|y(x, t)-y_{d}(x)\right|^{2} d x d t+\frac{1}{2} \int_{0}^{T} \int_{\omega}|u(x, t)|^{2} d x d t,
$$

subject to $(y, u) \in C\left([0, T] ; L^{2}(\Omega)\right) \times L^{2}\left(0, T ; L^{2}(\Omega)\right)$ satisfying the semilinear heat equation with a cubic nonlinearity

$$
\left\{\begin{array}{l}
y_{t}-\triangle y+y^{3}=\chi_{\omega} u \quad \text { in } \Omega \times(0, T) \\
y=0 \text { on } \partial \Omega \times(0, T) \\
y(0)=y_{0} \text { in } \Omega
\end{array}\right.
$$

This semilinear heat equation is well-posed. More precisely, given $y_{0} \in L^{2}(\Omega)$ and $u \in$ $L^{2}(\omega \times(0, T))$, there exists a unique solution $y \in C\left([0, T] ; L^{2}(\Omega)\right) \cap L^{2}\left(0, T ; H_{0}^{1}(\Omega)\right)$. Moreover, for each $T>0$, there exists at least one optimal solution $\left(y^{T}(\cdot), u^{T}(\cdot)\right)$. Meanwhile, there is an adjoint state $\lambda^{T}(\cdot)$ such that $\left(y^{T}(\cdot), \lambda^{T}(\cdot)\right)$ satisfies the optimality systems (cf., e.g., [28, section 3.1])

$$
\left\{\begin{array}{l}
y_{t}^{T}-\Delta y^{T}+\left(y^{T}\right)^{3}=\chi_{\omega} \lambda^{T} \quad \text { in } \Omega \times(0, T), \\
y^{T}=0 \quad \text { on } \partial \Omega \times(0, T), \\
y^{T}(0)=y_{0} \quad \text { in } \Omega, \\
\lambda_{t}^{T}+\Delta \lambda^{T}-3\left(y^{T}\right)^{2} \lambda^{T}=y^{T}-y_{d} \quad \text { in } \Omega \times(0, T), \\
\lambda^{T}=0 \text { on } \partial \Omega \times(0, T), \\
\lambda^{T}(T)=0 \text { in } \Omega,
\end{array}\right.
$$

and

$$
u^{T}(t)=\chi_{\omega} \lambda^{T}(t) \quad \text { for almost every } t \in(0, T)
$$

The corresponding static optimal control problem is

$$
\text { Minimize } \frac{1}{2} \int_{\Omega}\left|y(x)-y_{d}(x)\right|^{2} d x+\frac{1}{2} \int_{\omega}|u(x)|^{2} d x,
$$


subject to $(y, u) \in L^{2}(\Omega) \times L^{2}(\Omega)$ satisfying

$$
\left\{\begin{array}{l}
-\triangle y+y^{3}=\chi_{\omega} u \text { in } \Omega, \\
y=0 \text { on } \partial \Omega .
\end{array}\right.
$$

Obviously, any minimizer $\left(y_{s}, u_{s}\right)$ of this minimization problem satisfies

$$
\left\|y_{s}-y_{d}\right\|_{L^{2}(\Omega)}^{2}+\left\|u_{s}\right\|_{L^{2}(\omega)}^{2} \leqslant\left\|y_{d}\right\|_{L^{2}(\Omega)}^{2} .
$$

Moreover, for each minimizer $y_{s} \in H_{0}^{1}(\Omega)$, there exists $\lambda_{s} \in L^{2}(\Omega)$ such that

$$
\left\{\begin{array}{l}
-\triangle \lambda_{s}+3 y_{s}^{2} \lambda_{s}+y_{s}-y_{d}=0 \text { in } \Omega, \\
\lambda_{s}=0 \text { on } \partial \Omega .
\end{array}\right.
$$

In view of applying the elliptic regularity theory, we note that there exists $c=c(\Omega)>0$ such that

$$
\left\|y_{s}\right\|_{H_{0}^{1}(\Omega)}+\left\|\lambda_{s}\right\|_{H_{0}^{1}(\Omega)} \leqslant c\left\|y_{d}\right\|_{L^{2}(\Omega)} .
$$

We apply Theorem 1 with $X=L^{2}(\Omega), U=L^{2}(\omega), A=\triangle$ defined on the domain $D(A)=H^{2}(\Omega) \cap H_{0}^{1}(\Omega), f(y, u)=-y^{3}+\chi_{\omega} u$, and $f^{0}(y, u)=\frac{1}{2}\left\|y-y_{d}\right\|_{L^{2}(\Omega)}^{2}+$ $\frac{1}{2}\|u\|_{L^{2}(\omega)}^{2}$. In this case, we obtain by (2.11) that $H_{y u} H_{u u}^{-1} H_{u y}-H_{y y}$ is positive semidefinite (i.e., (2.8)) if the $L^{2}$-norm of $y_{d}$ is sufficiently small. Moreover,

$$
\mathcal{A}=A+O\left(\left\|y_{d}\right\|_{L^{2}(\Omega)}\right), \quad H_{\lambda u}=\chi_{\omega} I+O\left(\left\|y_{d}\right\|_{L^{2}(\Omega)}\right), \quad \mathcal{C}=I+O\left(\left\|y_{d}\right\|_{L^{2}(\Omega)}\right),
$$

where $I$ is the identity operator on $L^{2}(\Omega)$. Since the $C_{0}$ semigroup in $X$ generated by $A$ is exponentially stable, by the perturbation theory (see, e.g., [26, Chapter 3, Theorem 1.1])), the pairs $\left(\mathcal{A}, H_{\lambda u}\right)$ and $\left(\mathcal{A}^{*}, \mathcal{C}^{*}\right)$ are also exponentially stabilizable whenever the $L^{2}$-norm of $y_{d}$ is sufficiently small. From (2.11) again, we see that the condition (2.9) is also satisfied when $\left\|y_{d}\right\|_{L^{2}}$ and $\left\|y_{0}\right\|_{L^{2}}$ are small enough. Therefore, according to Theorem 1, the exponential turnpike property is valid provided that $\left\|y_{d}\right\|_{L^{2}}$ and $\left\|y_{0}\right\|_{L^{2}}$ are small enough. These smallness conditions are the same as in $[28]$.

Remark 4. In the above example, if the norm of the target $y_{d}$ is small, the operator $H_{y u} H_{u u}^{-1} H_{u y}-H_{y y}$ is not only positive semidefinite, but also it fills in the context of the exponential turnpike property of linear quadratic optimal control problems.

2.2. Periodic exponential turnpike for linear quadratic problems with periodic tracking trajectory. Let $X, U$, and $V$ be Hilbert spaces identified with their respective duals. As in the previous section, we first define the dynamical optimal control problem $\left(L Q^{T}\right)$, formulated as a linear-quadratic optimal control problem with a periodic tracking trajectory. Since the cost functional depends on $t$ in a periodic way, we replace the static optimal control problem with a periodic optimal control problem $\left(L Q^{\Pi}\right)$, whose solution yields the referred turnpike. The exponential turnpike result is then stated in Theorem 2 .

The dynamical optimal control problem $\left(L Q^{T}\right)$. Given any $y_{0} \in X$, we consider the linear control system

$$
\left\{\begin{array}{l}
\dot{y}(t)=A y(t)+B u(t), \quad t>0, \\
y(0)=y_{0}
\end{array}\right.
$$


where the operator $A: D(A) \subset X \rightarrow X$ generates a $C_{0}$ semigroup in $X$, and $B \in$ $L(U, X)$ is a linear bounded control operator.

Let $\Pi>0$ be a fixed positive real number. Let $y_{d}(\cdot) \in C([0,+\infty) ; X)$ and $u_{d}(\cdot) \in L_{l o c}^{2}(0,+\infty ; U)$ be two $\Pi$-periodic functions such that

$$
y_{d}(t+\Pi)=y_{d}(t), \quad u_{d}(t+\Pi)=u_{d}(t) \quad \text { for a.e. } t>0 .
$$

Let $C \in L(X, V)$ be a linear bounded observation operator, and let $Q \in L(X, X)$ be an invertible positive definite operator. For any $T>0$, we consider the optimal control problem

$$
\begin{aligned}
\left(L Q^{T}\right) \quad & \inf _{u(\cdot) \in L^{2}(0, T ; U)} J^{T}(u(\cdot)) \\
& =\frac{1}{2} \int_{0}^{T}\left(\left\|C\left(y(t)-y_{d}(t)\right)\right\|_{V}^{2}+\left\|Q^{1 / 2}\left(u(t)-u_{d}(t)\right)\right\|_{U}^{2}\right) d t,
\end{aligned}
$$

where $y(\cdot) \in C([0, T] ; X)$ is the solution of $(2.12)$ with the control $u(\cdot)$. In the literature, this minimization problem is usually referred to as a linear quadratic optimal problem with a periodic tracking trajectory.

The problem $\left(L Q^{T}\right)$ has a unique optimal solution $\left(y^{T}(\cdot), u^{T}(\cdot)\right)$. Moreover, following $[17,21]$ or $\left[24\right.$, Chapter 4 , Theorem 1.6]), there exists $\lambda^{T}(\cdot) \in C([0, T] ; X)$ such that

$$
\begin{cases}\dot{y}^{T}(t)=A y^{T}(t)+B Q^{-1} B^{*} \lambda^{T}(t)+B u_{d}(t), & y^{T}(0)=y_{0}, \\ \dot{\lambda}^{T}(t)=C^{*} C y^{T}(t)-A^{*} \lambda^{T}(t)-C^{*} C y_{d}(t), & \lambda^{T}(T)=0,\end{cases}
$$

in the mild sense along $[0, T]$ and

$$
u^{T}(t)=u_{d}(t)+Q^{-1} B^{*} \lambda^{T}(t), \text { a.e. } t \in[0, T] .
$$

The periodic optimal control problem $\left(L Q^{\Pi}\right)$. In the present case where the tracking terms in the cost functional depend on $t$, the turnpike property cannot anymore be captured by a corresponding static optimal control problem. Instead, we consider the periodic optimal control problem

$$
\begin{aligned}
\left(L Q^{\Pi}\right) \quad \inf & J^{\Pi}(y(\cdot), u(\cdot)) \\
= & \frac{1}{2} \int_{0}^{\Pi}\left(\left\|C\left(y(t)-y_{d}(t)\right)\right\|_{V}^{2}+\left\|Q^{1 / 2}\left(u(t)-u_{d}(t)\right)\right\|_{U}^{2}\right) d t,
\end{aligned}
$$

where $(y(\cdot), u(\cdot)) \in C([0, \Pi] ; X) \times L^{2}(0, \Pi ; U)$ is a mild solution of

$$
\left\{\begin{array}{l}
\dot{y}(t)=A y(t)+B u(t), \quad t \in[0, \Pi], \\
y(0)=y(\Pi) .
\end{array}\right.
$$

Existence and uniqueness for such periodic optimal control problems, as well as firstorder necessary conditions for optimality, have been widely studied in the existing literature (see, for instance, $[4,13,38]$ or $[24$, Chapter 4, Proposition 5.2] and references therein). Since the problem $\left(L Q^{\Pi}\right)$ is convex, it is well known that $\left(y^{\Pi}(\cdot), u^{\Pi}(\cdot)\right)$ is an optimal pair for $\left(L Q^{\Pi}\right)$ if and only if there exists an adjoint state $\lambda^{\Pi} \in C([0, \Pi] ; X)$ such that

$$
\begin{cases}\dot{y}^{\Pi}(t)=A y^{\Pi}(t)+B Q^{-1} B^{*} \lambda^{\Pi}(t)+B u_{d}(t), & y^{\Pi}(0)=y^{\Pi}(\Pi), \\ \dot{\lambda}^{\Pi}(t)=C^{*} C y^{\Pi}(t)-A^{*} \lambda^{\Pi}(t)-C^{*} C y_{d}(t), & \lambda^{\Pi}(0)=\lambda^{\Pi}(\Pi),\end{cases}
$$


in the mild sense along $[0, \Pi]$, and

$$
u^{\Pi}(t)=u_{d}(t)+Q^{-1} B^{*} \lambda^{\Pi}(t), \text { a.e. } t \in[0, \Pi] .
$$

The periodic exponential turnpike property.

TheOREm 2. Assume that the pair $(A, B)$ is exponentially stabilizable and that the pair $(A, C)$ is exponentially detectable. Then the following hold:

- The problem $\left(L Q^{\Pi}\right)$ has a unique solution $\left(y^{\Pi}(\cdot), u^{\Pi}(\cdot)\right)$, which has a unique extremal lift $\left(y^{\Pi}(\cdot), u^{\Pi}(\cdot), \lambda^{\Pi}(\cdot)\right)$ solution of (2.15) and (2.14). We extend it by $\Pi$-periodicity over $[0,+\infty)$.

- There exist positive constants $c$ and $\nu$ such that, for any $T>0$,

$$
\begin{aligned}
\left\|y^{T}(t)-y^{\Pi}(t)\right\|_{X}+\left\|u^{T}(t)-u^{\Pi}(t)\right\|_{U} \\
+\left\|\lambda^{T}(t)-\lambda^{\Pi}(t)\right\|_{X} \leqslant c\left(e^{-\nu t}+e^{-\nu(T-t)}\right)
\end{aligned}
$$

for every $t \in[0, T]$.

Remark 5. From the proof of the theorem, we infer the following explicit formulas in order to compute the optimal triple $\left(y^{\Pi}(\cdot), u^{\Pi}(\cdot), \lambda^{\Pi}(\cdot)\right)$. We claim that

$y^{\Pi}(t)=z(t)-E q(t), \quad \lambda^{\Pi}(t)=-P z(t)+(I+P E) q(t), \quad u^{\Pi}(t)=u_{d}(t)+Q^{-1} B^{*} \lambda^{\Pi}(t)$ for almost every $t \in[0, \Pi]$, where

- $P \in L(X, X)$ is the unique nonnegative self-adjoint operator solution of the operator algebraic Riccati equation

$$
A^{*} P+P A-P B Q^{-1} B^{*} P+C^{*} C=0,
$$

or equivalently,

$$
2\langle P A x, x\rangle_{X}-\left\langle P B Q^{-1} B^{*} P x, x\right\rangle_{X}+\langle C x, C x\rangle_{V}=0 \quad \forall x \in D(A) ;
$$

- $E \in L(X, X)$ is defined by

$$
E=-\int_{0}^{+\infty} S(t) B Q^{-1} B^{*} S(t)^{*} d t
$$

where $(S(t))_{t \geqslant 0}$ is the (exponentially stable) $C_{0}$ semigroup generated by the operator $A-B Q^{-1} B^{*} P$;

- $z(t)$ and $q(t)$ are the $\Pi$-periodic trajectories defined by

$$
\begin{aligned}
z(t)= & S(t)(I-S(\Pi))^{-1} \int_{0}^{\Pi} S(\Pi-\tau)\left((I+E P) B u_{d}(\tau)-E C^{*} C y_{d}(\tau)\right) d \tau \\
& +\int_{0}^{t} S(t-\tau)\left((I+E P) B u_{d}(\tau)-E C^{*} C y_{d}(\tau)\right) d \tau
\end{aligned}
$$

and

$$
\begin{aligned}
q(t)= & S(\Pi-t)^{*}\left(I-S(\Pi)^{*}\right)^{-1} \\
& \left.\times \int_{0}^{\Pi} S(\Pi-\tau)^{*}\left(-P B u_{d}(\Pi-\tau)+C^{*} C y_{d}(\Pi-\tau)\right)\right) d \tau \\
& +\int_{0}^{\Pi-t} S(\Pi-t-\tau)^{*}\left(-P B u_{d}(\Pi-\tau)+C^{*} C y_{d}(\Pi-\tau)\right) d \tau
\end{aligned}
$$

for every $t \in[0, \Pi]$. 
These facts are proved in section 3.1 (more specifically, see Lemma 3), as well as Theorem 2.

Remark 6 . Note that the periodicity of $y_{d}(\cdot)$ and $u_{d}(\cdot)$ does not play any role in the algebraic Riccati equation in Remark 5.

Remark 7. As can be seen from the proof of the theorem, the exponential decay constant $\nu$ in (2.16) can be characterized as the exponential stability rate for a $C_{0}$ semigroup resulting from the operator algebraic Riccati equation in Remark 5, and the constant $c$ in (2.16) is of the form $c_{1}\left(\left\|y_{0}-y^{\Pi}(0)\right\|_{X}+\left\|\lambda^{\Pi}(T)\right\|_{X}\right)$, where the constant $c_{1}$ does not depend on $y_{0}, y_{d}(\cdot)$, and $u_{d}(\cdot)$.

It follows from Theorem 2 that there exists $\eta>0$ such that, for any initial condition $y_{0}$, the optimal triple $\left(y^{T}(\cdot), u^{T}(\cdot), \lambda^{T}(\cdot)\right)$ solution of $\left(L Q^{T}\right)$ is exponentially close to the periodic optimal triple $\left(y^{\Pi}(\cdot), u^{\Pi}(\cdot), \lambda^{\Pi}(\cdot)\right)$ solution of $\left(L Q^{\Pi}\right)$ over the middle time interval $[\eta, T-\eta]$ whenever $T$ is large enough. Boundary layers may occur at $t=0$ and $t=T$ for the optimality system, and the exponential closeness is observed in the middle piece of optimal trajectories. This result means that, except at the extremities of the time frame, the optimal trajectory, as well as the optimal control and the associated adjoint state, is almost П-periodic. It is worth mentioning that similar results have been discussed in [3], [34], and [46, Chapter 6] for some finite-dimensional optimal control problems.

Example 2. Let $\Omega \subset \mathbb{R}^{n}(n \geqslant 1)$ be an open and bounded domain with a $C^{2}$ boundary, and let $\omega_{i} \subset \Omega, i=1,2$, be nonempty open subsets. Denote by $\chi_{\omega_{i}}$, $i=1,2$, the associated characteristic function. Let $y_{d} \in C\left([0,+\infty) ; L^{2}(\Omega)\right)$ be a periodic tracking trajectory, satisfying $y_{d}(t, \cdot)=y_{d}(t+1, \cdot)$ for any $t \geqslant 0$. Given any $T>0$, we consider the optimal control problem

$$
\text { Minimize } \frac{1}{2} \int_{0}^{T} \int_{\omega_{1}}\left|y(x, t)-y_{d}(x, t)\right|^{2} d x d t+\frac{1}{2} \int_{0}^{T} \int_{\omega_{2}}|u(x, t)|^{2} d x d t,
$$

over all possible $(y, u) \in C\left([0, T] ; L^{2}(\Omega)\right) \times L^{2}\left(0, T ; L^{2}(\Omega)\right)$ satisfying

$$
\left\{\begin{array}{l}
y_{t}-\triangle y+a(x) y=\chi_{\omega_{2}} u \quad \text { in } \Omega \times(0, T), \\
y=0 \text { on } \partial \Omega \times(0, T), \\
y(0)=y_{0} \quad \text { in } \Omega
\end{array}\right.
$$

where $a(\cdot) \in L^{\infty}(\Omega)$ and $y_{0} \in L^{2}(\Omega)$. We apply Theorem 2 with $X=U=V=L^{2}(\Omega)$, $A=\triangle-a(\cdot) I$ defined on the domain $D(A)=H^{2}(\Omega) \cap H_{0}^{1}(\Omega), B=\chi_{\omega_{2}} I$, and $C=\chi_{\omega_{1}} I$. Here, $I$ is the identity operator on $L^{2}(\Omega)$. Since the above heat equation with distributed control localized in $\omega_{i}, i=1,2$, is null controllable at any finite time (see, for instance, [50]), the pairs $(A, B)$ and $\left(A^{*}, C^{*}\right)$ are exponentially stabilizable (cf., e.g., [44, Theorem 3.3, p. 227]). Then, according to Theorem 2, we obtain that the corresponding periodic optimal control problem has a unique solution and that the optimal control problem under consideration has the periodic exponential turnpike property.

Example 3. Let $\Omega \subset \mathbb{R}^{n}(n \geqslant 1)$ be an open and bounded domain with a $C^{2}$ boundary, and let $\omega_{i} \subset \Omega, i=1,2$, be nonempty open subsets. Let $z_{d} \in$ $C\left([0,+\infty) ; L^{2}(\Omega)\right)$ be a periodic tracking trajectory, satisfying $z_{d}(t, \cdot)=z_{d}(t+1, \cdot)$ 
for any $t \geqslant 0$. Given any $T>0$, we consider the optimal control problem

$$
\text { Minimize } \frac{1}{2} \int_{0}^{T} \int_{\omega_{1}}\left|z_{t}(x, t)-z_{d}(x, t)\right|^{2} d x d t+\frac{1}{2} \int_{0}^{T} \int_{\omega_{2}}|u(x, t)|^{2} d x d t
$$

over all possible $(z, u) \in C\left([0, T] ; H_{0}^{1}(\Omega)\right) \cap C^{1}\left([0, T] ; L^{2}(\Omega)\right) \times L^{2}\left(0, T ; L^{2}(\Omega)\right)$ satisfying

$$
\left\{\begin{array}{l}
z_{t t}-\triangle z=\chi_{\omega_{2}} u \quad \text { in } \Omega \times(0, T), \\
z=0 \quad \text { on } \partial \Omega \times(0, T), \\
z(0)=z_{0}, \quad z_{t}(0)=z_{1} \quad \text { in } \Omega
\end{array}\right.
$$

where $z_{0} \in H_{0}^{1}(\Omega)$ and $z_{1} \in L^{2}(\Omega)$. Writing the wave equation as a first-order system, we apply Theorem 2 with $X=H_{0}^{1}(\Omega) \times L^{2}(\Omega), U=V=L^{2}(\Omega)$,

$$
\begin{gathered}
A=\left(\begin{array}{cc}
0 & I \\
\triangle & 0
\end{array}\right) \quad \text { defined on the domain } D(A)=\left(H^{2}(\Omega) \cap H_{0}^{1}(\Omega)\right) \times H_{0}^{1}(\Omega), \\
B=\left(\begin{array}{c}
0 \\
\chi_{\omega_{2}} I
\end{array}\right), \quad C=\left(\begin{array}{cc}
0 & \chi_{\omega_{1}} I
\end{array}\right)
\end{gathered}
$$

where $I$ the identity operator on $L^{2}(\Omega)$. Note that $A^{*}=-A$. It is well known (see [5]) that, under the assumption that $\left(\Omega, \omega_{i}\right), i=1,2$, satisfies the so-called geometric control condition, ${ }^{6}$ there exists $T_{0}>0$ such that the wave equation with the distributed control localized in $\omega_{i}, i=1,2$, is null controllable at any time $T>$ $T_{0}$. Therefore, the pairs $(A, B)$ and $\left(A^{*}, C^{*}\right)$ are exponentially stabilizable. Then, according to Theorem 2, this optimal control problem has the periodic exponential turnpike property if $T>T_{0}$.

2.3. Particular case: Tracking a point. Consider the linear quadratic optimal control problem $\left(L Q^{T}\right)$ of section 2.2. If $y_{d}(t)=y_{d} \in X$ and $u_{d}(t)=u_{d} \in U$ do not depend on $t$, then Theorem 1 can be applied and the referred turnpike is an optimal solution of the static optimal control problem

$$
\left(P_{s}\right) \quad \quad \inf J_{s}(y, u)=\frac{1}{2}\left(\left\|C\left(y-y_{d}\right)\right\|_{V}^{2}+\left\|Q^{1 / 2}\left(u-u_{d}\right)\right\|_{U}\right),
$$

over the set of all $(y, u) \in X \times U$ satisfying the constraint $A y+B u=0$. Since $\left(P_{s}\right)$ is a convex programming problem, it is well known that $\left(y_{s}, u_{s}\right) \in X \times U$ is an optimal solution of $\left(P_{s}\right)$ if and only if there exists an adjoint state $\lambda_{s} \in X$ (see, e.g., [17, 21] or [35, Chapter 6]) such that $u_{s}=u_{d}+Q^{-1} B^{*} \lambda_{s}$ and

$$
\left\{\begin{array}{l}
A y_{s}+B Q^{-1} B^{*} \lambda_{s}+B u_{d}=0 \\
C^{*} C y_{s}-A^{*} \lambda_{s}-C^{*} C y_{d}=0
\end{array}\right.
$$

Note that the optimal solution of the periodic optimal problem $\left(L Q^{\Pi}\right)$ coincides with that of the corresponding steady-state optimal control problem $\left(P_{s}\right)$ (i.e., $\left(y^{\Pi}(\cdot), u^{\Pi}(\cdot)\right)$ $\left.\equiv\left(y_{s}, u_{s}\right)\right)$. More precisely, we have the following result.

\footnotetext{
${ }^{6}$ The geometric control condition stipulates, roughly, that every generalized ray of geometrical optics that propagates at unit speed in $\Omega$ and reflects on its boundary should intersect $\omega_{i}$ within time $T$.
} 
Theorem 3. Assume that $(A, B)$ is exponentially stabilizable and $(A, C)$ is exponentially detectable. If $y_{d}(\cdot) \equiv y_{d} \in X$ and $u_{d}(\cdot) \equiv u_{d} \in U$, then there exist positive constants $c$ and $\nu$ such that for any $T>0$,

$$
\left\|y^{T}(t)-y_{s}\right\|_{X}+\left\|u^{T}(t)-u_{s}\right\|_{U}+\left\|\lambda^{T}(t)-\lambda_{s}\right\|_{X} \leqslant c\left(e^{-\nu t}+e^{-\nu(T-t)}\right)
$$

for every $t \in[0, T]$.

This result means that, in the linear quadratic framework, Theorem 1 holds true globally. It unifies and extends the exponential turnpike theorems established in $[27,37]$.

Remark 8. Similar to Remark 7, the exponential decay constant $\nu$ in (2.18) can be characterized as the exponential stability rate for a $C_{0}$ semigroup resulting from the operator algebraic Riccati equation in Remark 5, and the constant $c$ in (2.18) is of the form $c_{1}\left(\left\|y_{0}-y_{s}\right\|_{X}+\left\|\lambda_{s}\right\|_{X}\right)$, where the constant $c_{1}$ does not depend on $y_{0}, y_{d}$, and $u_{d}$. This means in particular that, even if $y_{0}=y_{s}$, the time depending optimal control does not coincide with the steady state one. This is because, in particular, the adjoint state of the steady state optimality system does not satisfy the terminal condition on the adjoint state of the evolution optimality system. In fact the size of $\lambda_{s}$ coincides with the distance from $\lambda_{s}$ to the terminal condition of the adjoint state, which is zero.

Remark 9. In the linear quadratic case, the operators $\mathcal{A}, H_{\lambda u}, \mathcal{C}$ in Theorem 1 , respectively, coincide with $A, B, C$ in Theorem 3. In fact, Theorem 3 is a global version of Theorem 1 in the linear quadratic case (that is, $\varepsilon=+\infty$ ).

2.4. Extensions to analytic parabolic systems with unbounded admissible control operators. The previous analysis in sections 2.1, 2.2, and 2.3 was restricted to bounded control operators. For instance, we were able to treat heat and wave equations with internal control, but not with a boundary control because then the control operator is unbounded in that case.

In this section, we generalize the results of Theorems 1, 2, and 3 to optimal control problems of analytic parabolic systems with unbounded admissible control operators.

As before, let $X$ and $U$ be two reflexive Hilbert spaces (which are identified with their dual spaces), and let $A: D(A) \subset X \rightarrow X$ generate a $C_{0}$ semigroup $(S(t))_{t \geqslant 0}$ on $X$. Denote by $A^{*}$ the adjoint operator associated with $A$, with the domain $D\left(A^{*}\right)$, and by $X_{-1}$ the dual of $D\left(A^{*}\right)$ with respect to the pivot space $X$. Then $(S(t))_{t \geqslant 0}$ can be extended into a $C_{0}$ semigroup on $X_{-1}$ (see [41, Proposition 2.10.4]). We say that $B \in L\left(U, X_{-1}\right)$ is an admissible control operator for $(S(t))_{t \geqslant 0}$ if there exists $\tau>0$ such that $\operatorname{Ran} \Psi_{\tau} \subset X$ (see, e.g., [41, Definition 4.2.1]), where

$$
\Psi_{\tau} u=\int_{0}^{\tau} S(\tau-t) B u(t) d t, \quad u \in L^{2}(0, \tau ; U) .
$$

First, as in section 2.1, for every $T>0$ and every $y_{0} \in X$, we consider the optimal control problem

$$
\left(O C P^{T}\right) \quad \inf _{u(\cdot) \in L^{2}(0, T ; U)} J^{T}(u(\cdot))=\int_{0}^{T} f^{0}(y(t), u(t)) d t,
$$

where $y(\cdot) \in C([0, T] ; X)$ is the mild solution of

$$
\left\{\begin{array}{l}
\dot{y}(t)=A y(t)+f(y(t))+B u \text { for a.e. } t \in[0, T], \\
y(0)=y_{0} .
\end{array}\right.
$$


With the same notation and assumptions as in section 2.1, we have the following exponential turnpike result (similar to Theorem 1). Note that in the case of semilinear control systems considered above, one has $H_{\lambda u}=B$.

TheOrem 4. We assume that $A$ generates an analytic semigroup $(S(t))_{t \geqslant 0}$ in $X$ and that $B \in L\left(U, X_{-1}\right)$ is an admissible control operator for $(S(t))_{t \geqslant 0}$. Under the assumptions of Theorem 1 , there exist positive constants $\varepsilon, \mu$, and $c$ such that for any $T>0$, if

$$
\left\|y_{0}-y_{s}\right\|_{X}+\left\|\lambda_{s}\right\|_{X} \leqslant \varepsilon
$$

then, for any optimal extremal triple $\left(y^{T}(\cdot), u^{T}(\cdot), \lambda^{T}(\cdot)\right)$ of $\left(O C P^{T}\right)$, we have the exponential turnpike property

$$
\left\|y^{T}(t)-y_{s}\right\|_{X}+\left\|u^{T}(t)-u_{s}\right\|_{U}+\left\|\lambda^{T}(t)-\lambda_{s}\right\|_{X} \leqslant c\left(e^{-\mu t}+e^{-\mu(T-t)}\right)
$$

for every $t \in[0, T]$.

Remark 10. Note that the operator $\mathcal{A}$ given by (2.7) is a perturbation of the generator $A$. It is already known that if $B$ is an admissible control operator for the $C_{0}$ semigroup generated by $A$, then $B$ is also an admissible control operator for the $C_{0}$ semigroup generated by $\mathcal{A}$ (cf. [41, Corollary 5.5.1]). Moreover, if $A$ generates an analytic semigroup $(S(t))_{t \geqslant 0}$ and if $B$ is an admissible control operator for $(S(t))_{t \geqslant 0}$, then by a standard perturbation result [26, Corollary 2.4, p. 81$]$, we can see that $\mathcal{A}$ also generates an analytic semigroup.

Second, with the same notation and assumptions introduced in section 2.2, for any $T>0$ and $y_{0} \in X$, we consider the optimal control problem $\left(L Q^{T}\right)$ by replacing the bounded control operator $B$ by an unbounded but admissible one. Then, we have the following two analogous results to Theorems 2 and 3.

Theorem 5. We assume that $A$ generates an analytic semigroup $(S(t))_{t \geqslant 0}$ in $X$ and that $B \in L\left(U, X_{-1}\right)$ is an admissible control operator for $(S(t))_{t \geqslant 0}$. We assume that the pair $(A, B)$ is exponentially stabilizable and that the pair $(A, C)$ is exponentially detectable. Then the following hold:

- The problem $\left(L Q^{\Pi}\right)$ has a unique solution $\left(y^{\Pi}(\cdot), u^{\Pi}(\cdot)\right)$, which has a unique extremal lift $\left(y^{\Pi}(\cdot), u^{\Pi}(\cdot), \lambda^{\Pi}(\cdot)\right)$. We extend it by $\Pi$-periodicity over $[0,+\infty)$.

- There exist positive constants $c$ and $\nu$ such that, for any $T>0$,

$$
\begin{aligned}
& \left\|y^{T}(t)-y^{\Pi}(t)\right\|_{X}+\left\|u^{T}(t)-u^{\Pi}(t)\right\|_{U} \\
& \quad+\left\|\lambda^{T}(t)-\lambda^{\Pi}(t)\right\|_{X} \leqslant c\left(e^{-\nu t}+e^{-\nu(T-t)}\right)
\end{aligned}
$$

for every $t \in[0, T]$.

Theorem 6. We assume that $A$ generates an analytic semigroup $(S(t))_{t \geqslant 0}$ in $X$ and that $B \in L\left(U, X_{-1}\right)$ is an admissible control operator for $(S(t))_{t \geqslant 0}$. We assume that $(A, B)$ is exponentially stabilizable and that $(A, C)$ is exponentially detectable. If $y_{d}(\cdot) \equiv y_{d} \in X$ and $u_{d}(\cdot) \equiv u_{d} \in U$, then there exist positive constants $c$ and $\nu$ such that for any $T>0$,

$$
\left\|y^{T}(t)-y_{s}\right\|_{X}+\left\|u^{T}(t)-u_{s}\right\|_{U}+\left\|\lambda^{T}(t)-\lambda_{s}\right\|_{X} \leqslant c\left(e^{-\nu t}+e^{-\nu(T-t)}\right)
$$

for every $t \in[0, T]$. 
Remark 11. Regarding the constants appearing in Theorems 4, 5, and 6, we could, respectively, have the same comments as in Remarks 2, 7, and 8.

We provide an examples below, concerning optimal control problems of the heat equation with Neumann boundary controls.

Example 4 . Let $\Omega \subset \mathbb{R}^{n}$ be a bounded domain with a smooth boundary $\partial \Omega$, and let $\Gamma \subset \partial \Omega$ be a nonempty open subset. Let $T>0, y_{0} \in L^{2}(\Omega)$ and $y_{d} \in$ $C\left([0,+\infty) ; L^{2}(\Omega)\right)$ be a periodic tracking trajectory, satisfying $y_{d}(t, \cdot)=y_{d}(t+1, \cdot)$ for any $t \geqslant 0$. Consider the optimal Neumann boundary control problem

$$
\inf _{u \in L^{2}\left(0, T ; L^{2}(\Gamma)\right)} \frac{1}{2} \int_{0}^{T}\left(\left\|y(t)-y_{d}(t)\right\|_{L^{2}(\Omega)}^{2}+\|u(t)\|_{L^{2}(\Gamma)}^{2}\right) d t,
$$

where $y \in C\left([0, T] ; L^{2}(\Omega)\right)$ is the solution of

$$
\begin{cases}y_{t}-\triangle y=0 & \text { in } \Omega \times(0, T) \\ \frac{\partial y}{\partial \nu}=u & \text { on } \Gamma \times(0, T) \\ y=0 & \text { on }(\partial \Omega \backslash \Gamma) \times(0, T) \\ y(0)=y_{0} & \text { in } \Omega\end{cases}
$$

Let $X=L^{2}(\Omega), U=L^{2}(\Gamma), V=L^{2}(\Omega), A=\triangle$ with $D(A)=\left\{y \in H^{2}(\Omega) \mid \frac{\partial y}{\partial \nu}=\right.$ 0 on $\partial \Omega\}, B=-\triangle N$, where $N: L^{2}(\partial \Omega) \rightarrow L^{2}(\Omega)$ is the Neumann map, ${ }^{7}$ and $C=I$ is the identity operator on $L^{2}(\Omega)$. Then, this optimal control problem can be reformulated as (see, e.g., [19, Chapter 3.3])

$$
\inf _{u \in L^{2}(0, T ; U)} \frac{1}{2} \int_{0}^{T}\left(\left\|C\left(y(t)-y_{d}(t)\right)\right\|_{V}^{2}+\|u(t)\|_{U}^{2}\right) d t,
$$

such that

$$
\dot{y}=A y+B u, \quad \text { for a.e. } t \in[0, T], y(0)=y_{0} .
$$

Furthermore, we actually have $B \in L\left(U,\left(D(A)^{1 / 4+\varepsilon}\right)^{\prime}\right.$ ) for every $\varepsilon>0$ (where $\left(D(A)^{1 / 4+\varepsilon}\right)^{\prime}$ is the dual space of $D(A)^{1 / 4+\varepsilon}$ with respect to the pivot space $X$ ), and thus $B$ is an admissible control operator for the analytic semigroup generated by $A$. Note that $(A, B)$ is exponentially stabilizable and that $(A, C)$ is automatically exponentially detectable when $C=I$. Therefore, according to Theorem 5 , the corresponding periodic optimal control problem has a unique solution, and the optimal control problem under consideration has the periodic exponential turnpike property.

2.5. A numerical simulation. In this section, we provide a simple example in order to numerically illustrate the periodic turnpike phenomenon in the finitedimensional case. Given any $T>0$, we consider the optimal control problem of minimizing the cost functional

$$
\frac{1}{2} \int_{0}^{T}\left((x(t)-\cos (2 \pi t))^{2}+(y(t)-\sin (2 \pi t))^{2}+u(t)^{2}\right) d t
$$

for the two-dimensional control system

$$
\dot{x}(t)=y(t), \quad \dot{y}(t)=u(t), \quad t \in(0, T),
$$

\footnotetext{
${ }^{7}$ The name "Neumann map" is due to the fact that $z=N u$ is the solution of the Neumann problem: $\Delta z=0$ in $\Omega$ and $\frac{\partial z}{\partial \nu}=u$ on $\partial \Omega$.
} 
with fixed initial condition $(x(0), y(0))=(0.1,0)$. Here, the target trajectories are 1-periodic. The explicit formulas for the expected periodic turnpike are given in Remark 5 and have been numerically computed with MATLAB. More precisely, to fit in the framework that has been developed previously, we set

$$
A=\left(\begin{array}{ll}
0 & 1 \\
0 & 0
\end{array}\right), \quad B=\left(\begin{array}{l}
0 \\
1
\end{array}\right), \quad C=Q=I, \quad u_{d} \equiv 0, \quad y_{d}(t)=\left(\begin{array}{c}
\cos (2 \pi t) \\
\sin (2 \pi t)
\end{array}\right),
$$

and then, using MATLAB, do the following:

- We solve the Riccati equation $A^{*} P+P A-P B B^{*} P+I=0$.

- We solve the Lyapunov equation $\left(A-B B^{*} P\right) E+E\left(A-B B^{*} P\right)^{*}-B B^{*}=0$.

- We set $\mathcal{T}=\left(\begin{array}{cc}I & -E \\ -P & I+P E\end{array}\right)$ and $S(t)=\exp \left(t\left(A-B B^{*} P\right)\right)$.

- We compute

$$
z(t)=-S(t)(I-S(1))^{-1} \int_{0}^{1} S(1-\tau) E y_{d}(\tau) d \tau-\int_{0}^{t} S(t-\tau) E y_{d}(\tau) d \tau
$$

and

$$
\begin{aligned}
q(t)= & S(1-t)^{*}\left(I-S(1)^{*}\right)^{-1} \int_{0}^{1} S(1-\tau)^{*} y_{d}(1-\tau) d \tau \\
& +\int_{0}^{1-t} S(1-t-\tau)^{*} y_{d}(1-\tau) d \tau
\end{aligned}
$$

for $t \in[0,1]$. This can be done by noting that $z(\cdot)$ and $q(\cdot)$ are solutions of some ordinary differential equations and by using numerical integration.

- Then, the reference turnpike trajectory and adjoint state are given by

$$
\left(\begin{array}{c}
(\bar{x}(t), \bar{y}(t)) \\
\left(\bar{\lambda}_{x}(t), \bar{\lambda}_{y}(t)\right)
\end{array}\right)=\mathcal{T}\left(\begin{array}{c}
z(t) \\
q(t)
\end{array}\right), \quad t \in[0,1] .
$$

The optimal extremal $\left(x(\cdot), y(\cdot), \lambda_{x}(\cdot), \lambda_{y}(\cdot), u(\cdot)\right)$, solution of the first-order optimality system derived from the Pontryagin maximum principle, has been computed in time $T=20$ by using a direct method of numerical optimal control (see [36]); more precisely, we have discretized the above optimal control problem using a Crank-Nicolson method and we have then used the automatic differentiation code AMPL (see [12]) combined with the optimization routine IpOpt (see [42]) on a standard desktop machine.

The turnpike property can be observed on Figure 1. As expected, except transient initial and final arcs, the extremal $\left(x(\cdot), y(\cdot), \lambda_{x}(\cdot), \lambda_{y}(\cdot)\right)$ (in blue) remains close to the periodic turnpike $\left(\bar{x}(\cdot), \bar{y}(\cdot), \bar{\lambda}_{x}(\cdot), \bar{\lambda}_{y}(\cdot)\right)$ (in red).

2.6. Conclusion and further comments. We have established the exponential turnpike property around an optimal steady-state for general nonlinear infinitedimensional optimal control problems under certain stability and smallness assumptions. We have then established the periodic exponential turnpike property for linear quadratic optimal control problems with periodic tracking trajectories, for which the turnpike is an optimal solution to a periodic optimal control problem. To the best of our knowledge, the latter result is new even in the finite-dimensional setting. Moreover, the optimal periodic solution has been explicitly characterized by means of a dichotomy transformation on the solutions of the operator algebraic Riccati and Lyapunov equations.

Some possible perspectives are in order. 


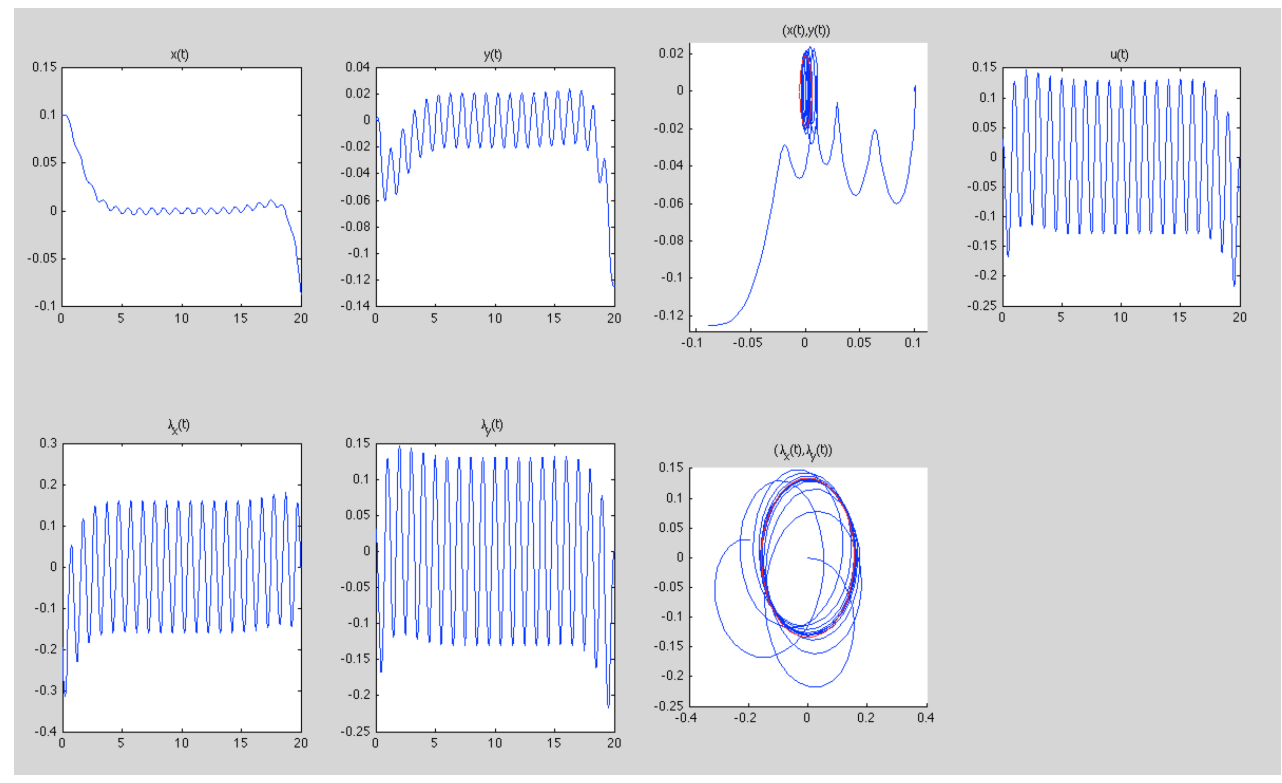

FIG. 1. Example of a periodic turnpike.

1. We believe that the periodic turnpike phenomenon appears in many concrete situations. It is, for instance, reminiscent of many biological processes (see, e.g., [15]). Then, obtaining a nonlinear version of our periodic turnpike theorem would certainly model many possible problems in life sciences. When linearizing the extremal system (derived from the Pontryagin maximum principle) around the optimal periodic trajectory, we obtain a linear time-periodic Hamiltonian system instead of an autonomous one. Identifying adequate hyperbolic properties is then an open problem. In a forthcoming paper, we will investigate the periodic turnpike phenomenon for optimal controls of general nonlinear time-periodic systems.

2. An open and challenging problem, probably much more difficult, is to remove the assumption of analyticity of the $C_{0}$ semigroup generated by $A$ in Theorems 4,5 , and 6 . To the best of our knowledge, the theory of algebraic Riccati equation with unbounded control operator for the hyperbolic case is incomplete so far (see, e.g., [20]). It seems to us that the general approach used in this paper cannot be developed and applied to this situation. There exist however particular situations where a turnpike result can be established: we refer to [16] and [51] for an exponential turnpike result for a one-dimensional wave equation with Neumann boundary control.

3. Although the results established in this paper are only in the framework of Hilbert spaces, the methodology used here is applicable to the more general reflexive Banach spaces, in which the Pontryagin maximum principle for optimal control problems is valid. Using the solvability of algebraic Riccati equation in Banach spaces (see, e.g., [18]) instead of that in Hilbert spaces, a similar procedure can be carried out with some slight modifications as what we have done in our analysis for the case of Hilbert spaces.

4. We emphasize that the final state $y(T)$ in the underlying optimal control problem is assumed to be free. This raises an obvious question: what happens if the final state is fixed, for instance, $y(T)=y_{1}$ ? In finite dimension, the 
exponential turnpike theorem for this question has been established in [37] under suitable Kalman rank condition. In infinite dimension, however, from our analysis there is a twofold difficulty for this question. On one hand, the Pontryagin maximum principle may fail in general. On the other hand, even if the Pontryagin maximum principle is valid under certain finite codimensional condition on the final state (cf. [24, Chapter 4, Theorem 1.6]), however we still do not know how to prove the invertibility of the Lyapunov operator $E$ constructed in (3.6). Indeed, it is generally not invertible. For example, when

$$
A=\left(\begin{array}{cc}
-1 & 0 \\
0 & -1
\end{array}\right), \quad B=\left(\begin{array}{l}
1 \\
0
\end{array}\right), \quad C=0, \quad Q=\left(\begin{array}{ll}
1 & 0 \\
0 & 1
\end{array}\right),
$$

one can easily check that $E$ is not invertible.

5. We have established our main results for optimal control problems not involving any state or control constraint. Indeed, the presence of constraints complicates the dynamics derived from the Pontryagin maximum principle, and then identifying hyperbolicity features may be very challenging. For instance, control constraints may promote chattering, i.e., an infinite number of control switchings over a compact time interval. Note that a turnpike phenomenon is suspected in [49], while chattering also occurs.

Consider, for instance, the linear quadratic optimal control problem $\left(L Q^{T}\right)$ considered in section 2.2. If we impose the simple control constraint $u \geqslant 0$, then the application of the Pontryagin maximum principle leads to the same extremal equations (2.13), and to the extremal control

$$
u(t)=\max \left(0, u_{d}(t)+Q^{-1} B^{*} \lambda^{T}(t)\right) .
$$

Since the extremal control is not smooth anymore, it is not clear how to analyze and use hyperbolicity properties of the extremal system, by linearization around the equilibrium corresponding to the optimal steady-state. Oscillations may indeed occur (with possible chattering as mentioned above) and make the dynamical study complex.

It is therefore challenging to consider the exponential turnpike property for optimal control problems with mixed state and/or control constraints, particularly including the time and norm optimal control problems for heat equations, at least, by using the approach developed in this paper, consisting of linearizing the extremal equations coming from the application of the Pontryagin maximum principle.

Instead, an interesting alternative consists of using dissipativity properties of the control system. We refer to [9] for investigating the exponential turnpike property for a class of strictly dissipative discrete-time systems in finite dimension. Even in the presence of constraints, the turnpike property may be analyzed within the viewpoint of strict dissipativity (see $[9,11,14,39]$ ). Such an analysis may reveal some relationships between strictly dissipativity and hyperbolicity, which are now two methodologies used in the literature to study the exponential turnpike property. In [39], we provide a comparison between these two approaches which yield results of different natures.

6. Our Theorem 1 has been proved under the smallness assumption (2.9), because our approach consists of linearizing the optimality system near the optimal steady-state that is an equilibrium point of the optimality system. Hence, this linearized system does not reflect what may happen far from this equilibrium point, and this is why the smallness condition (2.9) is then required in 
the proof. Establishing a global result, without such smallness assumptions, may certainly be done, but at the price of having a good knowledge of the global dynamics. Note that, in $[30,31]$, the authors study in a specific context the optimality status of several turnpikes that are in competition. Besides, dissipativity properties of the global dynamics (see $[9,11,14,39]$ ) may also be a route to deriving global turnpike properties.

7. Turnpike issues can be explored as well for shape optimization problems, by raising the question of whether optimal designs of a shape optimization problem for evolution systems approximate to those of an optimal steadystate one, as the time horizon is large enough (cf., e.g., [40]). We refer to [2] for an example of such a large time behavior for the two-phase optimal design for the heat equation, by using relaxation and homogenization. We notice that whether the admissible shapes depend on time or not makes a huge difference in the framework of shape optimization problems for evolution systems.

3. Proofs. For optimal control problems governed by infinite-dimensional evolution systems in which the Pontryagin maximum principle can be applied, we obtain a Hamiltonian system (two-point boundary value problem) coupling the optimal state and the associated adjoint state. In the proofs, we develop a dichotomy transformation acting on the solutions of the operator algebraic Riccati and Lyapunov type equations, in order to "decouple" the Hamiltonian system to a block-diagonal one, containing a contracting part (which is stable) and an expanding part (which is unstable). As a byproduct, we obtain a quantitative description of the limiting behavior, interpreted as the exponential turnpike property, of the optimal solutions of the original optimal control problem, as the time horizon is large enough.

Since the proofs of Theorems 2 and 3 are much easier than that of Theorem 1 , for the reader's convenience, we first give their proofs in section 3.1 and then we provide the proof of Theorem 1 in section 3.2. Finally, in section 3.3, we point out the key points and outline the proof of Theorem 6. Following the approach used in the proof of Theorem 6 , the proofs of Theorems 4 and 5 are almost the same as those of Theorems 1 and 2, respectively. Thus we omit their proofs and leave the details to the reader.

3.1. Proofs of Theorems 2 and 3. First, note that the extremal solution $\left(y_{s}, \lambda_{s}\right)$ of the problem $\left(P_{s}\right)$, solution of $(2.17)$, is an equilibrium point for the Hamiltonian system $(2.13)$ with $y_{d}(\cdot) \equiv y_{d}$ and $u_{d}(\cdot) \equiv u_{d}$. We are going to prove that this equilibrium is a saddle point for the system (2.13), thus yielding the turnpike property. Setting

$$
\delta y(t)=y^{T}(t)-y_{s}, \quad \delta \lambda(t)=\lambda^{T}(t)-\lambda_{s}, \quad t \in[0, T],
$$

we get from (2.13) and (2.17) that

$$
\frac{d}{d t}\left(\begin{array}{l}
\delta y(t) \\
\delta \lambda(t)
\end{array}\right)=M\left(\begin{array}{l}
\delta y(t) \\
\delta \lambda(t)
\end{array}\right), t \in[0, T]
$$

where $M: D(A) \times D\left(A^{*}\right) \rightarrow X \times X$ is the linear unbounded operator block defined by

$$
M=\left(\begin{array}{cc}
A & B Q^{-1} B^{*} \\
C^{*} C & -A^{*}
\end{array}\right) .
$$


Lemma 1. Assume that $(A, B)$ is exponentially stabilizable and that $(A, C)$ is exponentially detectable. Then $M$ is block-diagonalizable and boundedly invertible. Moreover, the system (3.2) can be decoupled by a bounded linear transformation.

Proof. It is well known from [44, Part IV, Chapter 4, Theorem 4.4, p. 241] that, under these assumptions in this lemma, the operator algebraic Riccati equation

$$
A^{*} P+P A-P B Q^{-1} B^{*} P+C^{*} C=0
$$

has a unique nonnegative self-adjoint operator solution $P \in L(X, X)$. Moreover, the operator $A-B Q^{-1} B^{*} P$ generates an exponentially stable $C_{0}$ semigroup $(S(t))_{t \geqslant 0}$, satisfying

$$
\|S(t)\|_{L(X, X)} \leqslant c e^{-\nu t}, \quad t \geqslant 0
$$

for some constants $c>0$ and $\nu>0$. As a consequence (see [44, Theorem 3.1, p. 222], for instance), the spectral abscissa of $A-B Q^{-1} B^{*} P$ satisfies

$$
\sup \left\{\operatorname{Re} \lambda \mid \lambda \in \sigma\left(A-B Q^{-1} B^{*} P\right)\right\} \leqslant-\nu<0,
$$

and thus the operator $A-B Q^{-1} B^{*} P$ is boundedly invertible. Since the semigroup $(S(t))_{t \geqslant 0}$ is exponentially stable, the Lyapunov integral operator $E \in L(X, X)$ given by

$$
E=-\int_{0}^{+\infty} S(t) B Q^{-1} B^{*} S(t)^{*} d t
$$

is well defined and is the solution of the operator Lyapunov equation (see also [44, Theorem 3.2, p. 226])

$$
\left(A-B Q^{-1} B^{*} P\right) E+E\left(A-B Q^{-1} B^{*} P\right)^{*}-B Q^{-1} B^{*}=0,
$$

or equivalently,

$$
2\left\langle\left(A-B Q^{-1} B^{*} P\right) E x, x\right\rangle_{X}-\left\langle B Q^{-1} B^{*} x, x\right\rangle_{X}=0 \quad \forall x \in D(A) .
$$

We now construct a dichotomy transformation in order to decouple the system (3.2), based on the linear and bounded operators $P$ and $E$. We first define two linear transformations on $X \times X$ by

$$
\mathcal{T}_{1}=\left(\begin{array}{ll}
I & 0 \\
P & I
\end{array}\right) \quad \text { and } \quad \mathcal{T}_{2}=\left(\begin{array}{cc}
I & 0 \\
-P & I
\end{array}\right)
$$

where $I$ is the identity operator on $X$. Note that

$$
\mathcal{T}_{1} \circ \mathcal{T}_{2}=\left(\begin{array}{ll}
I & 0 \\
0 & I
\end{array}\right)=\mathcal{T}_{2} \circ \mathcal{T}_{1}
$$

Since $P$ solves the Riccati equation (3.4), a straightforward computation shows that

$$
\mathcal{T}_{1} \circ M \circ \mathcal{T}_{2}=\left(\begin{array}{cc}
A-B Q^{-1} B^{*} P & B Q^{-1} B^{*} \\
0 & -\left(A-B Q^{-1} B^{*} P\right)^{*}
\end{array}\right) .
$$

Setting

$$
\left(\begin{array}{c}
v(t) \\
w(t)
\end{array}\right)=\mathcal{T}_{1}\left(\begin{array}{l}
\delta y(t) \\
\delta \lambda(t)
\end{array}\right), t \in[0, T]
$$


we infer from (3.2) and (3.8) that

$$
\frac{d}{d t}\left(\begin{array}{c}
v(t) \\
w(t)
\end{array}\right)=\left(\begin{array}{cc}
A-B Q^{-1} B^{*} P & B Q^{-1} B^{*} \\
0 & -\left(A-B Q^{-1} B^{*} P\right)^{*}
\end{array}\right)\left(\begin{array}{c}
v(t) \\
w(t)
\end{array}\right), \quad t \in[0, T]
$$

Now, we set

$$
\mathcal{T}_{3}=\left(\begin{array}{cc}
I & E \\
0 & I
\end{array}\right) \quad \text { and } \quad \mathcal{T}_{4}=\left(\begin{array}{cc}
I & -E \\
0 & I
\end{array}\right)
$$

Note that

$$
\mathcal{T}_{3} \circ \mathcal{T}_{4}=\left(\begin{array}{ll}
I & 0 \\
0 & I
\end{array}\right)=\mathcal{T}_{4} \circ \mathcal{T}_{3} .
$$

By performing the transformation

$$
\left(\begin{array}{c}
z(t) \\
q(t)
\end{array}\right)=\mathcal{T}_{3}\left(\begin{array}{c}
v(t) \\
w(t)
\end{array}\right), \quad t \in[0, T]
$$

we infer from (3.9) and (3.7) that

$$
\frac{d}{d t}\left(\begin{array}{c}
z(t) \\
q(t)
\end{array}\right)=\left(\begin{array}{cc}
A-B Q^{-1} B^{*} P & 0 \\
0 & -\left(A-B Q^{-1} B^{*} P\right)^{*}
\end{array}\right)\left(\begin{array}{l}
z(t) \\
q(t)
\end{array}\right), t \in[0, T] .
$$

Finally, we see that $M$ can be block-diagonalized by using the composition transformation $\mathcal{T}=\mathcal{T}_{3} \circ \mathcal{T}_{1}$ given by

$$
\mathcal{T}=\left(\begin{array}{cc}
I+E P & E \\
P & I
\end{array}\right)
$$

Since $A-B Q^{-1} B^{*} P$ and its adjoint operator are boundedly invertible, $M$ is boundedly invertible. This completes the proof.

Remark 12. Lemma 1 implies that the optimality system (2.17) has a unique solution.

Remark 13. In the finite-dimensional case, a dichotomy transformation similar to the one designed above has been used in [22, Lemma 2.5] and [33]. Here, we adapt this dichotomy transformation in the setting of infinite-dimensional Hilbert spaces. We also refer the reader to [43] for a different dichotomy transformation, which is, however, based on positive and negative definite solutions of the matrix algebraic Riccati equation.

Remark 14. The applications of dichotomy transformations for the Hamiltonian system, resulting from the Pontryagin maximum principle, are also well known in the numerical analysis of optimal control problems. For this issue we refer the reader to the brief paper [29]. Note that this uncoupling dichotomy transformation technique could be used as well for the numerical analysis of optimal control problems for partial differential equations.

The following stability estimate is inspired from [27, Lemma 3.5].

Lemma 2. Assume that $(A, B)$ is exponentially stabilizable and that $(A, C)$ is exponentially detectable. Then, there exists a constant $c>0$ independent of $T$ such that

$$
\|y(T)\|_{X}+\|\lambda(0)\|_{X} \leqslant c\left(\|y(0)\|_{X}+\|\lambda(T)\|_{X}\right)
$$


for any solution $(y(\cdot), \lambda(\cdot)) \in C([0, T] ; X) \times C([0, T] ; X)$ of the coupled system

$$
\left\{\begin{array}{l}
\dot{y}(t)=A y(t)+B Q^{-1} B^{*} \lambda(t), \\
\dot{\lambda}(t)=C^{*} C y(t)-A^{*} \lambda(t), \quad t \in[0, T] .
\end{array}\right.
$$

Proof. Since the pair $(A, C)$ is exponentially detectable, the pair $\left(A^{*}, C^{*}\right)$ is exponentially stabilizable, and thus there exists a bounded linear operator $K \in L(X, V)$ such that the $C_{0}$ semigroup generated by $A^{*}+C^{*} K$ is exponentially stable. Let $\varphi(\cdot) \in C([0, T] ; X)$ be the unique solution of

$$
\left\{\begin{array}{l}
\dot{\varphi}(t)=-\left(A^{*}+C^{*} K\right) \varphi(t), \quad t \in[0, T] \\
\varphi(T)=y(T)
\end{array}\right.
$$

It follows from the exponential decay of the $C_{0}$ semigroup that there is a constant $c_{1}>0$ (independent of $T$ ) such that

$$
\|\varphi(0)\|_{X} \leqslant c_{1}\|y(T)\|_{X}
$$

and

$$
\int_{0}^{T}\|\varphi(t)\|_{X}^{2} d t \leqslant c_{1}\|y(T)\|_{X}^{2}
$$

Multiplying by $\varphi(t)$ the first equation in (3.12) and integrating the result over $t \in$ $[0, T]$, we get

$$
\|y(T)\|_{X}^{2}=\langle y(0), \varphi(0)\rangle_{X}+\int_{0}^{T}\left(\left\langle B Q^{-1} B^{*} \lambda(t), \varphi(t)\right\rangle_{X}-\left\langle K^{*} C y(t), \varphi(t)\right\rangle_{X}\right) d t .
$$

By the Cauchy-Schwarz inequality and (3.14), we see that

$$
\begin{aligned}
& \int_{0}^{T}\left|\left\langle B Q^{-1} B^{*} \lambda(t), \varphi(t)\right\rangle_{X}\right| d t \\
& \quad \leqslant\left(\int_{0}^{T}\left\|B Q^{-1} B^{*} \lambda(t)\right\|_{X}^{2} d t\right)^{1 / 2}\left(\int_{0}^{T}\|\varphi(t)\|_{X}^{2} d t\right)^{1 / 2} \\
& \quad \leqslant c_{2}\|y(T)\|_{X}\left\|B Q^{-1 / 2}\right\|_{L(U, X)}\left(\int_{0}^{T}\left\|Q^{-1 / 2} B^{*} \lambda(t)\right\|_{U}^{2} d t\right)^{1 / 2}
\end{aligned}
$$

and

$$
\begin{aligned}
& \int_{0}^{T}\left|\left\langle K^{*} C y(t), \varphi(t)\right\rangle_{X}\right| d t \\
& \quad \leqslant\left\|K^{*}\right\|_{L(V, X)}\left(\int_{0}^{T}\|C y(t)\|_{V}^{2} d t\right)^{1 / 2}\left(\int_{0}^{T}\|\varphi(t)\|_{X}^{2} d t\right)^{1 / 2} \\
& \quad \leqslant c_{3}\left\|K^{*}\right\|_{L(V, X)}\|y(T)\|_{X}\left(\int_{0}^{T}\|C y(t)\|_{V}^{2} d t\right)^{1 / 2}
\end{aligned}
$$


These two inequalities, together with (3.15) and (3.13), imply that

$$
\|y(T)\|_{X}^{2} \leqslant c_{4}\left(\int_{0}^{T}\|C y(t)\|_{V}^{2}+\left\|Q^{-1 / 2} B^{*} \lambda(t)\right\|_{U}^{2} d t+\|y(0)\|_{X}^{2}\right)
$$

for some positive constant $c_{4}$ (independent of $T$ ).

Similarly, since the pair $(A, B)$ is exponentially stabilizable, we obtain from the second equation in (3.12) that

$$
\|\lambda(0)\|_{X}^{2} \leqslant c_{5}\left(\int_{0}^{T}\left\|Q^{-1 / 2} B^{*} \lambda(t)\right\|_{U}^{2}+\|C y(t)\|_{V}^{2} d t+\|\lambda(T)\|_{X}^{2}\right)
$$

for some positive constant $c_{5}$ (independent of $T$ ).

Let $c_{6}=\max \left(c_{4}, c_{5}\right)$. Next, multiplying by $\lambda(t)$ the first equation in (3.12) and by $y(t)$ the second equation in (3.12), and then integrating over $t \in[0, T]$, we get from the Cauchy-Schwarz inequality that

$$
\begin{aligned}
\int_{0}^{T} & \left.\left(\|C y(t)\|_{V}^{2}+\| Q^{-1 / 2} B^{*} \lambda(t)\right) \|_{U}^{2}\right) d t \\
& =\langle y(T), \lambda(T)\rangle_{X}-\langle y(0), \lambda(0)\rangle_{X} \\
& \leqslant\|\lambda(T)\|_{X}\|y(T)\|_{X}+\|y(0)\|_{X}\|\lambda(0)\|_{X} \\
& \leqslant c_{6}\|\lambda(T)\|_{X}^{2}+\frac{1}{4 c_{6}}\|y(T)\|_{X}^{2}+c_{6}\|y(0)\|_{X}^{2}+\frac{1}{4 c_{6}}\|\lambda(0)\|_{X}^{2} .
\end{aligned}
$$

This, along with (3.17) and (3.16), implies that

$$
\int_{0}^{T}\left(\|C y(t)\|_{V}^{2}+\left\|Q^{-1 / 2} B^{*} \lambda(t)\right\|_{U}^{2}\right) d t \leqslant c_{7}\left(\|y(0)\|_{X}^{2}+\|\lambda(T)\|_{X}^{2}\right)
$$

for some positive constant $c_{7}$ independent of $T$. Using (3.17) and (3.16) again, this leads to (3.11) and completes the proof.

Using Lemmas 1 and 2, we are now in position to prove Theorem 3.

Proof of Theorem 3. Let $\delta y(\cdot)$ and $\delta \lambda(\cdot)$ be defined by (3.1). By using the same dichotomy transformation

$$
\left(\begin{array}{l}
z(t) \\
q(t)
\end{array}\right)=\left(\begin{array}{cc}
I+E P & E \\
P & I
\end{array}\right)\left(\begin{array}{l}
\delta y(t) \\
\delta \lambda(t)
\end{array}\right), t \in[0, T]
$$

as in the proof of Lemma 1, we obtain a decoupled evolution system (3.10). Consequently, we have

$$
z(t)=S(t) z(0) \text { and } q(t)=S(T-t)^{*} q(T), t \in[0, T],
$$

where the $C_{0}$ semigroup $(S(t))_{t \geqslant 0}$ is generated by the operator $A-B Q^{-1} B^{*} P$ satisfying the exponential decay estimate (3.5), and $\left(S(t)^{*}\right)_{t \geqslant 0}$ is its adjoint $C_{0}$ semigroup.

Note that $u^{T}(t)-u_{s}=Q^{-1} B^{*} \delta \lambda(t)$ for a.e. $t \in[0, T]$. To derive the estimate (2.18), according to (3.18) and (3.19), it suffices to show that the norms of $z(0)$ and $q(T)$ have an upper bound which is independent of $T$. Note from (3.18) that

$$
z(0)=(I+E P) \delta y(0)+E \delta \lambda(0), \quad q(T)=P \delta y(T)+\delta \lambda(T) .
$$


Therefore, in order to complete the proof, it suffices to give an upper bound for $\|\delta y(T)\|_{X}$, as well as for $\|\delta \lambda(0)\|_{X}$, which follows from Lemma 2 . The theorem is proved.

Before turning to the proof of Theorem 2, by using Lemma 1 we are now in a position to formulate explicitly the optimal extremal triple $\left(y^{\Pi}(\cdot), u^{\Pi}(\cdot), \lambda^{\Pi}(\cdot)\right)$ for the periodic optimal control problem $\left(L Q^{\Pi}\right)$, thus proving the contents of Remark 5 .

Lemma 3. Under the assumptions of Theorem 2, the (unique) optimal П-periodic extremal triple $\left(y^{\Pi}(\cdot), \lambda^{\Pi}(\cdot), u^{\Pi}(\cdot)\right)$ of the problem $\left(L Q^{\Pi}\right)$ is given by

$y^{\Pi}(t)=z(t)-E q(t), \quad \lambda^{\Pi}(t)=-P z(t)+(I+P E) q(t), \quad u^{\Pi}(t)=u_{d}(t)+Q^{-1} B^{*} \lambda^{\Pi}(t)$

for almost every $t \in[0, \Pi]$. Here, $P$ and $E$ are accordingly linear bounded operators defined by (3.4) and (3.6), and $(z(t), q(t)), t \in[0, \Pi]$ are the periodic trajectories given by (3.25) and (3.26) below, respectively.

Proof. Using the dichotomy transformation already used in the proof of Lemma 1,

$$
\left(\begin{array}{c}
z(t) \\
q(t)
\end{array}\right)=\left(\begin{array}{cc}
I+E P & E \\
P & I
\end{array}\right)\left(\begin{array}{l}
y^{\Pi}(t) \\
\lambda^{\Pi}(t)
\end{array}\right), t \in[0, \Pi]
$$

we can uncouple the system (2.14) to

$$
\dot{z}(t)=\left(A-B Q^{-1} B^{*} P\right) z(t)+\left((I+E P) B u_{d}(t)-E C^{*} C y_{d}(t)\right), \quad t \in[0, \Pi],
$$

and

$$
\dot{q}(t)=-\left(A-B Q^{-1} B^{*} P\right)^{*} q(t)+\left(P B u_{d}(t)-C^{*} C y_{d}(t)\right), \quad t \in[0, \Pi] .
$$

Now, using the periodic boundary conditions, we are going to determine the initial data $z(0)$ and $q(T)$ for (3.21) and (3.22), respectively. It follows from (3.20) and from the periodic condition in (2.14) that

$$
z(0)=z(\Pi)
$$

By the Duhamel formula, we get from (3.21) that

$$
z(\Pi)=S(\Pi) z(0)+\int_{0}^{\Pi} S(\Pi-\tau)\left((I+E P) B u_{d}(\tau)-E C^{*} C y_{d}(\tau)\right) d \tau,
$$

where $(S(t))_{t \geqslant 0}$ is the $C_{0}$ semigroup generated by the operator $A-B Q^{-1} B^{*} P$ satisfying the exponential decay estimate (3.5). It follows from [4, Corollary 2.1] that the operator $I-S(\Pi)$ is boundedly invertible. Hence, we obtain from (3.23) and (3.24) that

$$
z(0)=(I-S(\Pi))^{-1} \int_{0}^{\Pi} S(\Pi-\tau)\left((I+E P) B u_{d}(\tau)-E C^{*} C y_{d}(\tau)\right) d \tau .
$$

Therefore, we get

$$
\begin{aligned}
z(t)= & S(t)(I-S(\Pi))^{-1} \int_{0}^{\Pi} S(\Pi-\tau)\left((I+E P) B u_{d}(\tau)-E C^{*} C y_{d}(\tau)\right) d \tau \\
& +\int_{0}^{t} S(t-\tau)\left((I+E P) B u_{d}(\tau)-E C^{*} C y_{d}(\tau)\right) d \tau, \quad t \in[0, \Pi] .
\end{aligned}
$$


Applying similar arguments to (3.22), we also obtain that

$$
\begin{aligned}
q(t)= & \left.S(\Pi-t)^{*}\left(I-S(\Pi)^{*}\right)^{-1} \int_{0}^{\Pi} S(\Pi-\tau)^{*}\left(-P B u_{d}(\Pi-\tau)+C^{*} C y_{d}(\Pi-\tau)\right)\right) d \tau \\
& +\int_{0}^{\Pi-t} S(\Pi-t-\tau)^{*}\left(-P B u_{d}(\Pi-\tau)+C^{*} C y_{d}(\Pi-\tau)\right) d \tau, \quad t \in[0, \Pi] .
\end{aligned}
$$

Noting from the transformation (3.20) that

$$
\left(\begin{array}{l}
y^{\Pi}(t) \\
\lambda^{\Pi}(t)
\end{array}\right)=\left(\begin{array}{cc}
I & -E \\
-P & I+P E
\end{array}\right)\left(\begin{array}{c}
z(t) \\
q(t)
\end{array}\right), \quad t \in[0, \Pi]
$$

the lemma follows.

We can now complete the proof of Theorem 2.

Proof of Theorem 2. Setting

$$
\delta y(t)=y^{T}(t)-y^{\Pi}(t), \quad \delta \lambda(t)=\lambda^{T}(t)-\lambda^{\Pi}(t), \quad \delta u(t)=u^{T}(t)-u^{\Pi}(t), \quad t \in[0, T],
$$

we get from (2.13) and (2.14) that

$$
\left\{\begin{array}{l}
\frac{d \delta y(t)}{d t}=A \delta y(t)+B Q^{-1} B^{*} \delta \lambda(t), \quad t \in[0, T], \\
\frac{d \delta \lambda(t)}{d t}=C^{*} C \delta y(t)-A^{*} \delta \lambda(t), \quad t \in[0, T]
\end{array}\right.
$$

with the terminal conditions

$$
\delta y(0)=y_{0}-y^{\Pi}(0), \quad \delta \lambda(T)=-\lambda^{\Pi}\left(T-\left[\frac{T}{\Pi}\right] \Pi\right) .
$$

Here, $[x]$ denotes the largest integer less than or equal to $x$. Using the dichotomy transformation

$$
\left(\begin{array}{c}
v(t) \\
w(t)
\end{array}\right)=\left(\begin{array}{cc}
I+E P & E \\
P & I
\end{array}\right)\left(\begin{array}{l}
\delta y(t) \\
\delta \lambda(t)
\end{array}\right), t \in[0, T]
$$

where $P$ and $E$ are accordingly given by (3.4) and (3.6), we transform the system (3.27) to

$$
\frac{d}{d t}\left(\begin{array}{c}
v(t) \\
w(t)
\end{array}\right)=\left(\begin{array}{cc}
A-B Q^{-1} B^{*} P & 0 \\
0 & -\left(A-B Q^{-1} B^{*} P\right)^{*}
\end{array}\right)\left(\begin{array}{c}
v(t) \\
w(t)
\end{array}\right), \quad t \in[0, T] .
$$

Therefore,

$$
v(t)=S(t) v(0) \text { and } w(t)=S(T-t)^{*} w(T), t \in[0, T],
$$

where $(S(t))_{t \geqslant 0}$ is the exponentially stable $C_{0}$ semigroup generated by $A-B Q^{-1} B^{*} P$. In particular, by (3.28), we have

$$
v(0)=(I+E P) \delta y(0)+E \delta \lambda(0) \quad \text { and } \quad w(T)=\delta \lambda(T)+P \delta y(T) .
$$


By Lemma 2, we infer that the stability estimate

$$
\|\delta y(T)\|_{X}+\|\delta \lambda(0)\|_{X} \leqslant c\left(\|\delta y(0)\|_{X}+\|\delta \lambda(T)\|_{X}\right)
$$

holds true for some positive constant $c$ independent of $T$. This estimate, together with (3.30), (3.29), as well as the bounded invertibility of the dichotomy transformation (3.28), lead to the estimate

$$
\|\delta y(t)\|_{X}+\|\delta \lambda(t)\|_{X} \leqslant c\left(\|\delta y(0)\|_{X}+\|\delta \lambda(T)\|_{X}\right)\left(e^{-\nu t}+e^{-\nu(T-t)}\right) \quad \forall t \in[0, T]
$$

for some positive constants $c$ and $\nu$ independent of $T$. The theorem is proved.

3.2. Proof of Theorem 1. We follow the arguments of the proofs of Lemmas 1, 2 and of Theorem 3, as well as those of the proof of [37, Theorem 1].

According to the assumptions, the Hamiltonian $H$ is twice continuously Fréchet differentiable in $X \times X \times U$, and thus for any $(y, \lambda, u) \in X \times X \times U$, there exists a constant $R_{0}>0$ such that, for any $0<R<R_{0}$, the asymptotic expansion formula

$$
\begin{aligned}
& H_{\star}(y+\delta y, \lambda+\delta \lambda, u+\delta u)-H_{\star}(y, \lambda, u) \\
& \quad=H_{\star y}(y, \lambda, u) \delta y+H_{\star \lambda}(y, \lambda, u) \delta \lambda+H_{\star u}(y, \lambda, u) \delta u+o(\delta y, \delta \lambda, \delta u)
\end{aligned}
$$

holds for any small perturbation $(\delta y, \delta \lambda, \delta u)$ verifying

$$
\|\delta y\|_{X}+\|\delta \lambda\|_{X}+\|\delta u\|_{U} \leqslant R
$$

Here the symbol $\star$ stands for the Fréchet derivative of $H$ with respect to the variable $y$ or $\lambda$ or $u$, and $o(\delta h)$ is the remaining higher-order terms with respect to $\delta h$. by

We start by defining perturbations of $\left(y^{T}(\cdot), \lambda^{T}(\cdot), u^{T}(\cdot)\right)$ with respect to $\left(y_{s}, \lambda_{s}, u_{s}\right)$,

$$
\delta y(t)=y^{T}(t)-y_{s}, \quad \delta \lambda(t)=\lambda^{T}(t)-\lambda_{s}, \quad \delta u(t)=u^{T}(t)-u_{s}, \quad t \in[0, T] .
$$

Under the assumptions of the theorem, we make the following a priori hypotheses:

$$
\begin{array}{ll}
\text { (i). } & \|(\delta y(t), \delta \lambda(t), \delta u(t))\|_{X \times X \times U} \leqslant R \text { for a.e. } t \in[0, T] ; \\
\text { (ii). } & \int_{0}^{T}\left(\|\delta y(t)\|_{X}^{2}+\|\delta \lambda(t)\|_{X}^{2}\right) d t \leqslant R
\end{array}
$$

where the positive constant $R$ is sufficiently small. These two hypotheses will be verified a posteriori at the end by an appropriate choice of smallness constraint (2.9). First, it follows from (2.3) and (2.6) that, at the point $\left(y_{s}, \lambda_{s}, u_{s}\right)$,

$$
H_{u y} \delta y(t)+H_{u \lambda} \delta \lambda(t)+H_{u u} \delta u(t)+o(\delta y(t), \delta \lambda(t), \delta u(t))=0 \text { for a.e. } t \in[0, T] .
$$

Since $H_{u u}^{-1}$ is bounded, we get

$$
\delta u(t)=-H_{u u}^{-1}\left(H_{u y} \delta y(t)+H_{u \lambda} \delta \lambda(t)\right)+o(\delta y(t), \delta \lambda(t)) .
$$

Therefore, using (2.7) and (2.8), we infer from (2.2), (2.5), and (3.33) that

$$
\frac{d}{d t}\left(\begin{array}{l}
\delta y(t) \\
\delta \lambda(t)
\end{array}\right)=\left(\begin{array}{cc}
\mathcal{A} & -H_{\lambda u} H_{u u}^{-1} H_{u \lambda} \\
\mathcal{C}^{*} \mathcal{C} & -\mathcal{A}^{*}
\end{array}\right)\left(\begin{array}{l}
\delta y(t) \\
\delta \lambda(t)
\end{array}\right)+o(\delta y(t), \delta \lambda(t))
$$


with the two-point boundary conditions

$$
\delta y(0)=y_{0}-y_{s}, \quad \delta \lambda(T)=-\lambda_{s}
$$

Note that the principal part of (3.34) has the same structure as the operator block $M$ given by (3.3). As already mentioned, in comparison with the proof of Theorem 3, the difficulty is here to deal carefully with the higher-order remaining terms in (3.34).

Next, by using arguments similar to those in the proof of Lemma 1, we are going to uncouple the principal part of the linearized system (3.34). Let $\mathcal{P} \in L(X, X)$ be the unique nonnegative self-adjoint operator solution of the operator algebraic Riccati equation (see the same reasonings for (3.4))

$$
\mathcal{A}^{*} \mathcal{P}+\mathcal{P} \mathcal{A}+\mathcal{C}^{*} \mathcal{C}+\mathcal{P} H_{\lambda u} H_{u u}^{-1} H_{u \lambda} \mathcal{P}=0
$$

Moreover, the operator $\mathcal{A}+H_{\lambda u} H_{u u}^{-1} H_{u \lambda} \mathcal{P}$ generates an exponentially stable $C_{0}$ semigroup $(\mathcal{S}(t))_{t \geqslant 0}$ in $X$, i.e.,

$$
\|\mathcal{S}(t)\|_{L(X, X)} \leqslant c_{1} e^{-\nu t} \text { for } t \geqslant 0
$$

for some positive constants $c_{1}$ and $\nu$. We then define the linear bounded selfadjoint operator on $X$

$$
\mathcal{E}=\int_{0}^{+\infty} \mathcal{S}(t) H_{\lambda u} H_{u u}^{-1} H_{u \lambda} \mathcal{S}(t)^{*} d t
$$

We can check that it satisfies the Lyapunov equation (see the same reasonings for $(3.7))$

$$
\left(\mathcal{A}+H_{\lambda u} H_{u u}^{-1} H_{u \lambda} \mathcal{P}\right) \mathcal{E}+\mathcal{E}\left(\mathcal{A}+H_{\lambda u} H_{u u}^{-1} H_{u \lambda} \mathcal{P}\right)^{*}+H_{\lambda u} H_{u u}^{-1} H_{u \lambda}=0 .
$$

Using the dichotomy transformation

$$
\left(\begin{array}{c}
v(t) \\
w(t)
\end{array}\right)=\left(\begin{array}{cc}
I+\mathcal{E} \mathcal{P} & \mathcal{E} \\
\mathcal{P} & I
\end{array}\right)\left(\begin{array}{l}
\delta y(t) \\
\delta \lambda(t)
\end{array}\right), \quad t \in[0, T]
$$

we transform the system (3.34) to

$\frac{d}{d t}\left(\begin{array}{c}v(t) \\ w(t)\end{array}\right)=\left(\begin{array}{cc}\mathcal{A}+H_{\lambda u} H_{u u}^{-1} H_{u \lambda} \mathcal{P} & 0 \\ 0 & -\left(\mathcal{A}+H_{\lambda u} H_{u u}^{-1} H_{u \lambda} \mathcal{P}\right)^{*}\end{array}\right)\left(\begin{array}{c}v(t) \\ w(t)\end{array}\right)+o(v(t), w(t))$.

Solving the first equation in forward time and the second equation in backward time, and using (3.36), we get

$$
\|v(t)\|_{X}+\|w(t)\|_{X} \leqslant 4 c_{1}\left(e^{-\nu t / 2}\|v(0)\|_{X}+e^{-\nu(T-t) / 2}\|w(T)\|_{X}\right)
$$

for every $t \in[0, T]$.

The remainder of the proof consists of determining the values $\left(\|v(0)\|_{X},\|w(T)\|_{X}\right)$ from the terminal conditions (3.35). We first claim that the inequality

$$
\|\delta y(T)\|_{X}+\|\delta \lambda(0)\|_{X} \leqslant c_{2}\left(\left\|y_{0}-y_{s}\right\|_{X}+\left\|\lambda_{s}\right\|_{X}\right)+o(R)
$$

holds for some constant $c_{2}$ independent of $T$. (The proof of this claim is postponed 
to the end.) It follows from the transformation (3.37) that

$$
v(0)=(I+\mathcal{E P}) \delta y(0)+\mathcal{E} \delta \lambda(0), \quad w(T)=\mathcal{P} \delta y(T)+\delta \lambda(T) .
$$

Hence, we infer from (3.37), (3.38), and (3.39) that

$$
\|\delta y(t)\|_{X}+\|\delta \lambda(t)\|_{X} \leqslant\left(c_{3}\left(\left\|y_{0}-y_{s}\right\|_{X}+\left\|\lambda_{s}\right\|_{X}\right)+o(R)\right)\left(e^{-\nu t / 2}+e^{-\nu(T-t) / 2}\right)
$$

for every $t \in[0, T]$ with some constant $c_{3}$ independent of $T$. This, together with (3.33), leads to a similar estimate for $\delta u(\cdot)$. Therefore, there exists $\varepsilon>0$ such that the estimates (3.32) hold true whenever the inequality (2.9) holds. As a consequence of (3.40), the exponential turnpike property (2.10) is proved.

Finally, let us prove the claim (3.39), which is analogous to that in Lemma 2. For the first equation in $(3.34)$, since $\left(\mathcal{A}^{*}, \mathcal{C}^{*}\right)$ is exponentially detectable, by the same reasoning as for (3.16), there exists a $c_{0}>0$ independent of $T$ such that

$$
\|\delta y(T)\|_{X}^{2} \leqslant c_{0}\left(\int_{0}^{T}\left(\|\mathcal{C} \delta y(t)\|_{V}^{2}+\left\|H_{u u}^{-1 / 2} H_{u \lambda} \delta \lambda(t)\right\|_{U}^{2}\right) d t+\|\delta y(0)\|_{X}^{2}\right)+o(R) .
$$

Similarly, we obtain

$$
\|\delta \lambda(0)\|_{X}^{2} \leqslant c_{0}\left(\int_{0}^{T}\left(\left\|H_{u u}^{-1 / 2} H_{u \lambda} \delta \lambda(t)\right\|_{U}^{2}+\|\mathcal{C} \delta y(t)\|_{V}^{2}\right) d t+\|\delta \lambda(T)\|_{X}^{2}\right)+o(R) .
$$

Multiplying by $\delta \lambda(t)$ the first equation in (3.34) and by $\delta y(t)$ the second equation in (3.34), and then integrating over $t \in[0, T]$, we get that

$$
\begin{aligned}
& \int_{0}^{T}\left(\left\|H_{u u}^{-1 / 2} H_{u \lambda} \delta \lambda(t)\right\|_{U}^{2}+\|\mathcal{C} \delta y(t)\|_{V}^{2}\right) d t \\
& \quad \leqslant\|\delta \lambda(T)\|_{X}\|\delta y(T)\|_{X}+\|\delta y(0)\|_{X}\|\delta \lambda(0)\|_{X}+o(R) \\
& \quad \leqslant \sigma\left(\|\delta \lambda(0)\|_{X}^{2}+\|\delta y(T)\|_{X}^{2}\right)+\frac{1}{4 \sigma}\left(\|\delta y(0)\|_{X}^{2}+\|\delta \lambda(T)\|_{X}^{2}\right)+o(R)
\end{aligned}
$$

for any real number $\sigma>0$. This, together with (3.41) and (3.42), implies that

$$
\begin{aligned}
\int_{0}^{T} & \left(\left\|H_{u u}^{-1 / 2} H_{u \lambda} \delta \lambda(t)\right\|_{U}^{2}+\|\mathcal{C} \delta y(t)\|_{V}^{2}\right) d t \\
\leqslant & 2 c_{0} \sigma \int_{0}^{T}\left(\left\|H_{u u}^{-1 / 2} H_{u \lambda} \delta \lambda(t)\right\|_{U}^{2}+\|\mathcal{C} \delta y(t)\|_{V}^{2}\right) d t \\
\quad & +\left(\frac{1}{4 \sigma}+2 c_{0} \sigma\right)\left(\|\delta y(0)\|_{X}^{2}+\|\delta \lambda(T)\|_{X}^{2}\right)+o(R) .
\end{aligned}
$$

Choosing $\sigma=\frac{1}{4 c_{0}}$, we get that

$\int_{0}^{T}\left(\left\|H_{u u}^{-1 / 2} H_{u \lambda} \delta \lambda(t)\right\|_{U}^{2}+\|\mathcal{C} \delta y(t)\|_{V}^{2}\right) d t \leqslant\left(2 c_{0}+1\right)\left(\|\delta y(0)\|_{X}^{2}+\|\delta \lambda(T)\|_{X}^{2}\right)+o(R)$.

This, along with (3.35), (3.41), and (3.42), implies and completes the proof of the claim (3.39). 
Remark 15. The algebraic Riccati and Lyapunov equations involved in the proof of Theorem 1 coincide with those in the linear case (i.e., the proof of Theorem 3).

3.3. Proof of Theorem 6. Compared with the proof of Theorem 3 (which is the case of bounded control operators), the new difficulty here is the solvability of the operator algebraic Riccati equation and the operator Lyapunov equation, with unbounded admissible control operators. In the following, we mainly demonstrate how to overcome this difficulty and give a resolution.

More precisely, as seen in the proof of Theorem 3, there are two key ingredients to construct the dichotomy transformation:

(K1). There exists a solution $P \in L(X, X)$ to the operator algebraic Riccati equation

$$
A^{*} P+P A-P B Q^{-1} B^{*} P+C^{*} C=0 .
$$

(K2). There exists a solution $E \in L(X, X)$ to the operator Lyapunov equation

$$
\left(A-B Q^{-1} B^{*} P\right) E+E\left(A-B Q^{-1} B^{*} P\right)^{*}-B Q^{-1} B^{*}=0 .
$$

Observe that, once (K1) and (K2) have been solved, by following the same approach as in the proof of Theorem 3, we obtain Theorem 6 . Hereafter, we show that (K1) and (K2) can indeed be solved when the unbounded control operator $B$ is admissible for an analytic semigroup generated by $A$. We will finish the proof of Theorem 6 whenever they are done.

We first show that (K1) is valid. In fact, since $B$ is an admissible control operator for the $C_{0}$ semigroup generated by $A$, by [41, Proposition 4.4.6], we have $B \in L\left(U, X_{-1 / 2-\varepsilon}\right)$ for every $\varepsilon>0$. Then, under the assumptions of this theorem, one can easily verify that all assumptions of [19, Theorems 2.2.1 and 2.2.2, p. 125-127] are satisfied. Thus, we have the following facts about the algebraic Riccati equation for analytic parabolic systems:

(G1). There exists a nonnegative and self-adjoint operator $P \in L(X, X)$ satisfying the operator algebraic Riccati equation

$$
A^{*} P+P A-P B Q^{-1} B^{*} P+C^{*} C=0 .
$$

(G2). The operator $B^{*} P$ is bounded from $X$ to $U$, i.e., $B^{*} P \in L(X, U)$.

(G3). The operator $A-B Q^{-1} B^{*} P$ generates an exponentially stable $C_{0}$ semigroup $\left(S_{P}(t)\right)_{t \geqslant 0}$, satisfying

$$
\left\|S_{P}(t)\right\|_{L(X, X)} \leqslant c e^{-\nu t}, \quad t \geqslant 0,
$$

for some constants $c>0$ and $\nu>0$.

As a consequence, (G1) guarantees that (K1) is true.

Second, we prove that (K2) is also valid. In fact, by the duality between admissible observation and control operators (cf., e.g., [41, Theorem 4.4.3]), we obtain that $B^{*}$ is an admissible observation operator for the $C_{0}$ semigroup generated by $A^{*}$. It follows from (G2) that $P B \in L(U, X)$. Then, by the perturbation theory of admissible observation (see, e.g., [41, Theorem 5.4.2]), we have that $B^{*}$ is indeed an admissible observation operator for $\left(S_{P}(t)^{*}\right)_{t \geqslant 0}$. Because $\left(S_{P}(t)^{*}\right)_{t \geqslant 0}$ is moreover exponentially stable (see (G3) above), we deduce from [41, Remark 4.3.5] that $B^{*}$ is also an infinite- 
time admissible observation operator for $\left(S_{P}(t)^{*}\right)_{t \geqslant 0}$, i.e., there exists a constant $c>0$ (independent of $T$ ) such that

$$
\int_{0}^{+\infty}\left\|B^{*} S_{P}(t)^{*} z\right\|_{U}^{2} d t \leqslant c\|z\|_{X}^{2} \quad \forall z \in D\left(A^{*}\right) .
$$

From [41, Theorem 5.1.1], we find that there exists a linear and bounded operator $E \in L(X, X)$ satisfying the following operator Lyapunov equation

$$
\left(A-B Q^{-1} B^{*} P\right) E+E\left(A-B Q^{-1} B^{*} P\right)^{*}-B Q^{-1} B^{*}=0 .
$$

Thus, (K2) is also true. This completes the proof.

Acknowledgment. The authors wish to thank the anonymous referees for their careful reading and helpful comments to improve the presentation of this paper in a significant way, especially for the suggestion of studying the case of unbounded admissible control operators (see section 2.4).

\section{REFERENCES}

[1] B. D. O. Anderson And P. V. Kokotovic, Optimal control problems over large time intervals, Automatica J. IFAC, 23 (1987), pp. 355-363.

[2] G. Allaire, A. MÜnch, And F. Periago, Long time behavior of a two-phase optimal design for the heat equation, SIAM J. Control Optim., 48 (2010), pp. 5333-5356.

[3] Z. ARTSTEIN AND A. LeIZAROWITZ, Tracking periodic signals with the overtaking criterion, IEEE Trans. Automat. Control, 30 (1985), pp. 1123-1126.

[4] V. Barbu and N. H. Pavel, Periodic optimal control in Hilbert space, Appl. Math. Optim., 33 (1996), pp. 169-188.

[5] C. Bardos, G. Lebeau, And J. Rauch, Sharp sufficient conditions for the observation, control, and stabilization of waves from the boundary, SIAM J. Control Optim., 30 (1992), pp. 10241065.

[6] P. Cardaliaguet, J. M. Lasry, P. L. Lions, and A. Porretta, Long time average of mean field games with a nonlocal coupling, SIAM J. Control Optim., 51 (2013), pp. 3558-3591.

[7] D. A. Carlson, A. Haurie, and A. Jabrane, Existence of overtaking solutions to infinitedimensional control problems on unbounded time intervals, SIAM J. Control Optim., 25 (1987), pp. 1517-1541.

[8] D. A. Carlson, A. B. Haurie, and A. Leizarowitz, Infinite Horizon Optimal Control, 2nd ed., Springer, New York, 1991.

[9] T. Damm, L. Grüne, M. Stieler, and K. Worthmann, An exponential turnpike theorem for dissipative discrete time optimal control problems, SIAM J. Control Optim., 52 (2014), pp. 1935-1957.

[10] R. Dorfman, P. A. Samuelson, And R. Solow, Linear Programming and Economic Analysis, McGraw-Hill, New York, 1958.

[11] T. Faulwasser, M. Korda, C. N. Jones, and D. Bonvin, On turnpike and dissipativity properties of continuous-time optimal control problems, Automatica J. IFAC, 81 (2017), pp. 297-304.

[12] R. Fourer, D. M. Gay, And B. W. Kernighan, AMPL: A Modeling Language for Mathematical Programming, 2nd ed., Duxbury Press, Pacific Grove, CA, 2002.

[13] E. GILBerT, Optimal periodic control: A general theory of necessary conditions, SIAM J. Control Optim., 15 (1977), pp. 717-746.

[14] L. GRÜNE AND M. MÜLLER, On the relation between strict dissipativity and turnpike properties, Systems Control Lett., 90 (2016), pp. 45-53.

[15] F. Grognard, A. R. Akhmetzhanov, and O. Bernard, Periodic Optimal Control for Biomass Productivity Maximization in a Photobioreactor Using Natural Light, Inria research report 7929, 2012.

[16] M. Gugat, E. Trélat, And E. Zuazua, Optimal Neumann control for the $1 D$ wave equation: Finite horizon, infinite horizon, boundary tracking terms and the turnpike property, Systems Control Lett., 90 (2016), pp. 61-70.

[17] K. Ito And K. Kunisch, Lagrange Multiplier Approach to Variational Problems and Applications, Adv. Des. Control 15, SIAM, Philadelphia, 2008. 
[18] S. Koshkin, Positive semigroups and algebraic Riccati equations in Banach spaces, Positivity, 20 (2016), pp. 541-563.

[19] I. Lasiecka and R. Triggiani, Control Theory for Partial Differential Equations: Volume 1, Abstract Parabolic Systems: Continuous and Approximation Theories, Cambridge University Press, Cambridge, 2000.

[20] I. Lasiecka and R. Triggiani, Control Theory for Partial Differential Equations: Volume 2, Abstract Hyperbolic-Like Systems Over a Finite Time Horizon: Continuous and Approximation Theories, Cambridge University Press, Cambridge, 2000.

[21] J. L. Lions, Optimal Control for Systems Governed by Partial Differential Equations, Springer, New York, 1971.

[22] D. L. LuKES, Optimal regulation of nonlinear dynamical systems, SIAM J. Control, 7 (1969), pp. $75-100$.

[23] H. Lou And W. Wang, Turnpike Properties of Optimal Relaxed Control Problems, preprint.

[24] X. Li And J. Yong, Optimal Control Theory for Infinite-Dimensional Systems. Systems and Control: Foundations and Applications, Birkhäuser Boston, Boston, 1995.

[25] L. W. McKenzie, Turnpike theorems for a generalized Leontief model, Econometrica, 31 (1963), pp. $165-180$.

[26] A. PAZY, Semigroups of Linear Operators and Applications to Partial Differential Equations, Springer, New York, 1983.

[27] A. Porretta And E. ZuAzuA, Long time versus steady state optimal control, SIAM J. Control Optim., 51 (2013), pp. 4242-4273.

[28] A. Porretta AND E. ZuAzuA, Remarks on long time versus steady state optimal control, in Mathematical Paradigms of Climate Science. Springer, New York, 2016, pp. 67-89.

[29] A. V. RaO And K. D. Mease, Dichotomic basis approach to solving hypersensitive optimal control problems, Automatica J. IFAC, 35 (1999), pp. 633-642.

[30] A. RAPAPORT AND P. CARTIGNY, Turnpike theorems by a value function approach, ESAIM Control Optim. Calc. Var., 10 (2004), pp. 123-141.

[31] A. RAPAPORT AND P. CARTIGNY, Competition between most rapid approach paths: Necessary and sufficient conditions, J. Optim. Theory Appl., 124 (2005), pp. 1-27.

[32] R. T. RockAfEllar, Saddle points of Hamiltonian systems in convex problems of Lagrange, J. Optim. Theory Appl., 12 (1973), pp. 367-390.

[33] N. Saкамото, Analysis of the Hamilton-Jacobi equation in nonlinear control theory by symplectic geometry, SIAM J. Control Optim., 40 (2002), pp. 1924-1937.

[34] P. A. Samullson, The periodic turnpike theorem, Nonlinear Anal., 1 (1976), pp. 3-13.

[35] F. Tröltzsch, Optimal Control of Partial Differential Equations: Theory, Methods and Applications, Grad. Stud. Math. 112, AMS, Providence, RI, 2010.

[36] E. Trélat, Contrôle Optimal: Théorie \& Applications, Vuibert, Collection "Mathématiques Concrètes," 2005.

[37] E. TRÉlat and E. Zuazua, The turnpike property in finite-dimensional nonlinear optimal control, J. Differential Equations, 258 (2015), pp. 81-114.

[38] E. TrÉlat, L. WANG, AND Y. Zhang, Impulse and sampled-data optimal control of heat equations, and error estimates, SIAM J. Control Optim., 54 (2016), pp. 2787-2819.

[39] E. TrÉlat And C. Zhang, Integral and Measure-Turnpike Properties for Infinite-Dimensional Optimal Control Systems, preprint, arXiv:1705.02762, 2017.

[40] E. Trélat, C. Zhang, and E. Zuazua, Optimal shape design for $2 D$ heat equations in large time, Pure Appl. Funct. Anal., 3 (2018), pp. 255-269.

[41] M. TuCSNAK And G. Weiss, Observation and Control for Operator Semigroups, Springer, New York, 2009.

[42] A. Wächter AND L. T. Biegler, On the implementation of an interior-point filter line-search algorithm for large-scale nonlinear programming, Math. Program., 106 (2006), pp. 25-57.

[43] R. R. Wilde and P. V. Kokotovic, A dichotomy in linear control theory, IEEE Trans. Automat, Control, 17 (1972), pp. 382-383.

[44] J. ZABCZyK, Mathematical Control Theory: An Introduction. Systems and Control: Foundations and Applications, Birkhäuser Boston, Boston, 1992.

[45] M. Zanon, L. Grüne, And M. Dienl, Periodic optimal control, dissipativity and MPC, IEEE Trans. Automat. Control, 62 (2017), pp. 2943-2949.

[46] A. J. Zaslavski, Turnpike Properties in the Calculus of Variations and Optimal Control, Vol. 80, Springer, New York, 2006.

[47] A. J. Zaslavski, Turnpike Phenomenon and Infinite Horizon Optimal Control, Springer Optim. Appl. 99, Springer, New York, 2014.

[48] A. J. Zaslavski, Turnpike Theory of Continuous-Time Linear Optimal Control Problems, Springer Optim. Appl. 104, Springer, New York, 2015. 
[49] J. Zhu, E. Trélat, And M. Cerf, Minimum time control of the rocket attitude reorientation associated with orbit dynamics, SIAM J. Control Optim., 54 (2016), pp. 391-422.

[50] E. Zuazua, Controllability and observability of partial differential equations: Some results and open problems, in Handbook of Differential Equations: Evolutionary Differential Equations, Vol. 3, Elsevier Science, New York, 2006, pp. 527-621.

[51] E. ZuAzuA, Large time control and turnpike properties for wave equations, Annu. Rev. Control, 44 (2017), pp. 199-210. 